\title{
Mitofusin 2 attenuates the histone acetylation at collagen IV promoter in diabetic nephropathy
}

\author{
Xuhua Mi, Wanxin Tang, Xiaolei Chen, Fei Liu and Xiaohong Tang
}

Division of Nephrology, West China Hospital, Sichuan University, Chengdu, China

Correspondence should be addressed to W Tang

Email

kidney123@163.com

\begin{abstract}
Extracellular matrix (ECM) increase in diabetic nephropathy (DN) is closely related to mitochondrial dysfunction. The mechanism of protective function of mitofusin 2 (Mfn2) for mitochondria remains largely unknown. In this study, the molecular mechanisms for the effect of $M f n 2$ on mitochondria and subsequent collagen IV expression in DN were investigated. Ras-binding-deficient mitofusin 2 (Mfn2-Ras $(\Delta)$ ) were overexpressed in rat glomerular mesangial cells, and then the cells were detected for mitochondrial morphology, cellular reactive oxygen species (ROS), mRNA and protein expression of collagen IV with advanced glycation end-product (AGE) stimulation. Preliminary results reveal that the mitochondrial dysfunction and the increased synthesis of collagen IV after AGE stimulation were reverted by $\operatorname{Mfn} 2-\operatorname{Ras}(\Delta)$ overexpression. Bioinformatical computations were performed to search transcriptional factor motifs in the promoter region of collagen IV. Three specific regions for TFAP2A binding were identified, followed by validation with chromatin immunoprecipitation experiments. Knocking down TFAP2A significantly decreased the TF binding in the first two regions and the gene expression of collagen IV. Furthermore, results reveal that $M f n 2-\operatorname{Ras}(\Delta)$ overexpression significantly mitigated TFAP2A binding and also reverted the histone acetylation at Regions 1 and 2 after AGE stimulation. In streptozotocin-induced diabetic rats, Mfn2-Ras( $\Delta)$ overexpression also ameliorated glomerular mesangial lesions with decreased collagen IV expression, accompanied by decreased acetylation and TFAP2A binding at Region 1. In conclusion, this study highlights the pathway by which mitochondria affect the histone acetylation of gene promoter and provides a new potential therapy approach for DN.
\end{abstract}

Journal of Molecular Endocrinology (2016) 57, 233-249

\section{Introduction}

More than 347 million people suffer from diabetes worldwide (Danaei et al. 2011). Diabetic nephropathy (DN) is a serious complication of diabetes with poor prognosis and the most common cause of end-stage renal disease (Atkins \& Zimmet 2010). However, the mechanism by which DN arises is yet to be elucidated even though there is an urgent need to identify an

\author{
Key Words \\ - Mitofusin 2 \\ - histone \\ - acetylation \\ - collagen \\ - diabetic nephropathy
}

effective early intervention for DN. One of the hallmarks of DN is the expansion of glomerular mesangial area for increased synthesis of extracellular matrix (ECM), which is mainly composed of collagen IV and V, fibronectin and laminin, among others (Mene et al. 1989, Haneda et al. 1991). Several recent studies have shown substantial increase in the mesangial content of collagen IV induced 
by high glucose levels in mesangial cells (Whiteside et al. 2009, Li et al. 2013).

Emerging evidence indicates that oxidative stress serves as a critical pathogenic process in the development of DN (Onozato et al. 2002, Koya et al. 2003). Reactive oxygen species (ROS), which is mainly produced by mitochondria, is a significant factor causing oxidative stress in numerous pathological processes. Mitochondrial dysfunction in diabetes leads to overproduction of ROS, which has a major effect on disease progression (Brownlee 2001, 2005, Dugan et al. 2013). In addition, increasing evidence indicates that ECM accumulation in $\mathrm{DN}$ is closely associated with oxidative stress and mitochondrial dysfunction (Pozzi et al. 2009, Ribaldo et al. 2009). However, the underlying mechanism that explains the relationship remains unclear.

Mitofusin 2 (Mfn2), a dynamic regulatory protein found in the mitochondria, is critical for the maintenance of normal mitochondrial function in mammalian cells (Bach et al. 2003). Mfn2 is a multifunctional protein that promotes mitochondrial fusion (Anton et al. 2013), activates Ras-MAPK signaling pathway via its Ras-binding site (77th to 91th amino acid of Mfn2, N-DVKGYLSKVRGISEV-C) (de Brito \& Scorrano 2009), regulates mitochondrial transport (Misko et al. 2010) and maintains the stability of mitochondrial DNA (Vielhaber et al. 2013). Both basic and clinical studies suggest that Mfn2 is involved in mitochondrial biogenesis and metabolism in diabetes (Hernandez-Alvarez et al. 2010, Zorzano et al. 2010). In our previous study, overexpression of wild-type Mfn2 attenuated numerous pathological changes in kidney structure and function associated with diabetes in rats, including repression of collagen IV synthesis (Tang et al. 2012). However, the mechanism by which overexpression of $M f n 2$ affected the expression of pathogenic genes in $\mathrm{DN}$ remained unclear.

Abnormal gene transcription is a response to extracellular stimulation signals and is regulated by cis-regulatory elements, such as promoters, enhancers, silencers and insulators (Smith \& Shilatifard 2014). Among these elements, promoters perform a central function in activating gene transcription. The initial step in gene expression involves chromatin remodeling in the promoter region, which is mainly regulated by histone acetylation or methylation (Jenuwein \& Allis 2001, Kouzarides 2007). Important clues for the involvement of histone modifications in the regulation of pathologic genes that are associated with DN have emerged gradually in recent years. Previously, in endothelial cells cultured in high glucose, the prolonged upregulation of p65 with sustained changes in H3 lysine-4 monomethylation was implicated (El-Osta et al. 2008). In uninephrectomized $\mathrm{db} / \mathrm{db}$ mice, a model of non-insulin-dependent diabetes mellitus, H3K9 and H3K23 acetylation and H3K4 dimethylation significantly increased with severe glomerulosclerosis (Sayyed et al. 2010). The epigenetic characteristic of the promoter is enriched with histone 3 lysine- 4 trimethylation (H3K4me3), and the active form is enriched with histone 3 lysine-27 acetylation (H3K27ac) (Jin et al. 2011, Zhou et al. 2011). Findings of such epigenetic changes have offered a new framework for understanding the pathogenesis of $\mathrm{DN}$.

Increasing evidence indicates that ROS can directly induce epigenetic modification, consequently affecting gene expression. For instance, insulin alters the acetylation of histone $\mathrm{H} 3$ by enhancing ROS production under hyperglycemic conditions (Kabra et al. 2009). The level of H4K12 histone acetylation is significantly increased by superoxide overproduction in porcine oocytes during aging (Cui et al. 2011). In rat hepatocytes, oxidative stress induced by ethanol significantly stimulates histone $\mathrm{H} 3$ acetylation (Choudhury et al. 2010). To our knowledge, how ROS regulates key genes in DN at transcriptional level has yet to be extensively studied.

Elucidation of molecular mechanisms underlying DN at the transcriptional level is critical for understanding the pathogenesis of DN. In this study, we report that overexpression of Ras-binding-deficient mitofusin 2 $(M f n 2-\operatorname{Ras}(\Delta))$ attenuates AGE-dependent mitochondrial fragmentation and ROS production under conditions of diabetes in rat mesangial cells. We also found that overexpression of $M f n 2-\operatorname{Ras}(\Delta)$ has effects on histone acetylation and transcriptional factor (TF) binding at specific regions near the collagen IV transcriptional start site (TSS), which may be responsible for the increased expression of collagen IV in DN. In addition, epigenetic changes were also validated in rat DN models. Our results provide some new insights into the complex molecular pathogenic mechanisms of DN and identify potential avenues for early intervention of DN.

\section{Methods}

\section{Cell culture}

Rat mesangial cells were obtained from Texas Health Science Center and cultured in Dulbecco's modified Eagle's medium (DMEM) (Gibco) supplemented with 17\% fetal bovine serum (FBS) (Gibco) at $37^{\circ} \mathrm{C}$ in $5 \% \mathrm{CO}_{2}$ in a

Published by Bioscientifica Ltd. 
humidified incubator. The cells were grown in complete medium for further validation experiments at $80 \%$ confluence after synchronization.

\section{Preparation of advanced glycation end-product (AGE) proteins}

AGE proteins were synthesized as described previously (Oldfield et al. 2001). In brief, $10 \mathrm{mg} / \mathrm{mL}$ bovine serum albumin (BSA) was incubated with $0.5 \mathrm{M}$ D-glucose in $0.4 \mathrm{M}$ phosphate buffer at $37^{\circ} \mathrm{C}$ for 12 weeks under sterile conditions. Unincorporated sugars were then removed through dialysis against phosphate-buffered saline (PBS). Control non-glycated BSA was prepared by incubation without glucose. Glycation was assessed by characteristic fluorescence (excitation of $370 \mathrm{~nm}$ and emission of $440 \mathrm{~nm}$ ) with an 8- to 10-fold increase in fluorescence of AGE-BSA in comparison with BSA.

\section{Construction of $M f n 2$ gene vectors and transfection}

The rat Mfn2 mRNA sequence (NM_130894.4) was obtained from the NCBI website. The $M f n 2-\operatorname{Ras}(\Delta)$ sequence was subjected to Ras-binding region deletion. The target gene sequences were then subcloned between BamHI and AgeI restriction sites of the GV287 vector (element sequence: Ubi-MCS-3FLAG-SV40-EGFP). After amplification, the positive clone was confirmed by sequencing (GeneChem, Shanghai, China). Afterward, the purified reconstructed vectors combined with lentivirus (GeneChem) were used for transfection. In brief, rat glomerular mesangial cells (GMCs) were seeded in six-well plates in entire DMEM medium (Gibco) under normal culture condition. When cell growth reached $30-50 \%$ confluency, both $1 \times 10^{8} \mathrm{TU} / \mathrm{mL}$ lentivirus containing $20 \mu \mathrm{L}$ of reconstructed vectors and $1 \mu \mathrm{L}$ of $10 \mathrm{mg} / \mathrm{mL}$ Polybrene were added. After $8 \mathrm{~h}$, the culture liquid was replaced with fresh medium.
A
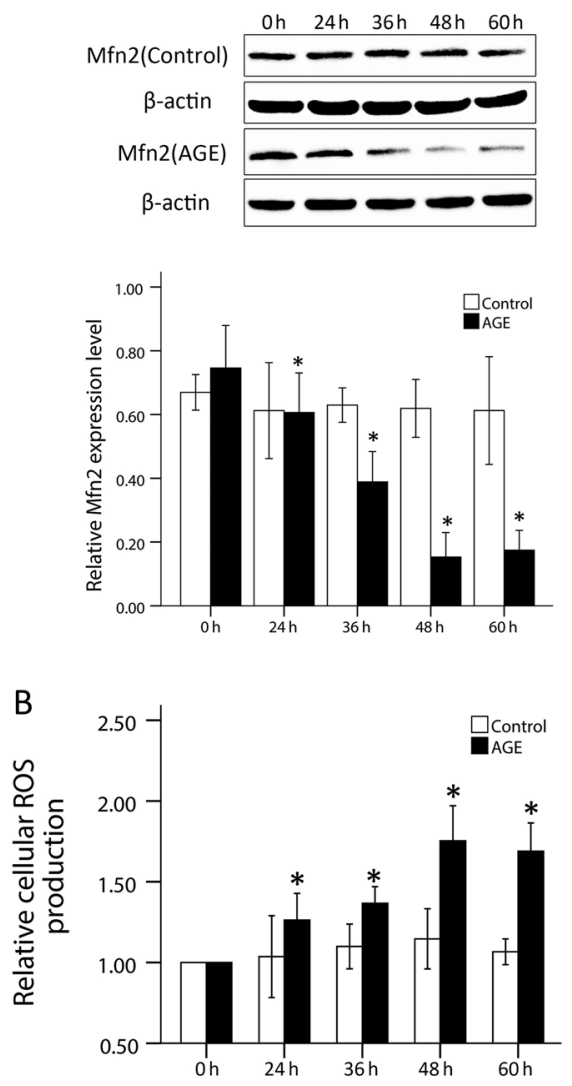

C

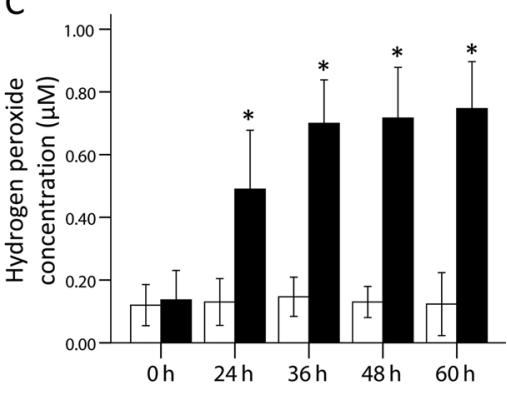

D

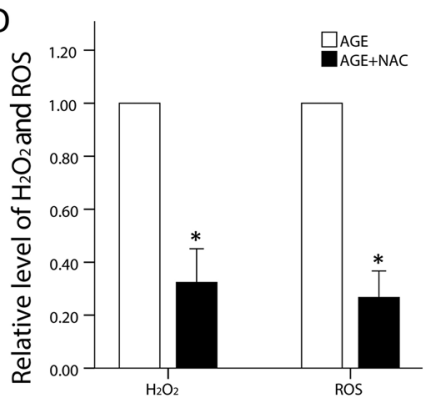

$E$

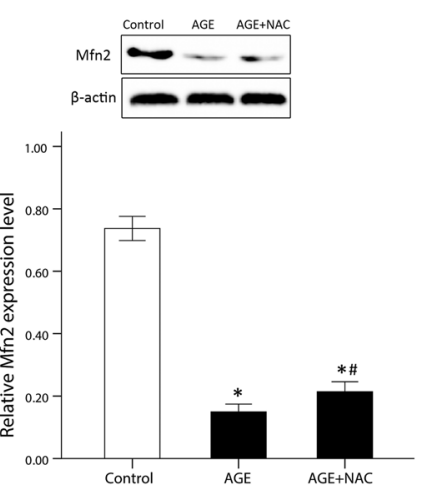

Figure 1

Downregulation of mitofusin 2 and increased production of cellular ROS and hydrogen peroxide induced by AGE in GMCs. GMCs were exposed to control BSA or AGE-BSA at the concentration of $40 \mu \mathrm{M}$. (A) The expression of mfn2 protein over time in GMCs under control BSA or AGE stimulation by Western blotting. $\beta$-actin was used as an internal control. ${ }^{*} P<0.05$ vs control group. (B) The relative production of cellular ROS over time under control BSA or AGE stimulation. ${ }^{*} P<0.05$ vs control at $0 \mathrm{~h}$. (C) The hydrogen peroxide concentration changed over time under AGE stimulation. ${ }^{*} P<0.05$ vs control group. (D) ROS and hydrogen peroxide relative levels in the presence of NAC under AGE stimulation. ${ }^{*} P<0.05$ vs AGE group at $48 \mathrm{~h}$. (E) The expression of $\mathrm{mfn} 2$ protein under AGE stimulation or ROS blocking by Western blotting. The samples were pretreated with NAC $(5 \mathrm{mM})$ for $2 \mathrm{~h}$ before the addition of AGE in AGE + NAC group. $\beta$-actin was used as internal control. ${ }^{*} P<0.05$ by $t$-test vs control group; ${ }^{*} P<0.05$ by $t$-test vs AGE group. Data are mean \pm S.E.M. of three separate experiments. http://jme.endocrinology-journals.org DOI: 10.1530/JME-16-0031
๑) 2016 Society for Endocrinology Printed in Great Britain 
The transfected GMCs were assessed for transfection efficiency and harvested for sub-culture after $72 \mathrm{~h}$.

\section{Co-immunoprecipitation}

GMC lysates from various groups were used for co-immunoprecipitation as described previously (Koshiba et al. 2004, Zhang et al. 2009). In brief, cell lysates were first cleared with 50\% protein A-agarose (Santa Cruz Biotechnology) and were then incubated with rabbit polyclonal antibody against $m f n 2$ protein (Thermo Scientific) for $1 \mathrm{~h}$ at $4^{\circ} \mathrm{C}$, followed by incubation with protein A-agarose overnight at $4^{\circ} \mathrm{C}$. The antigenantibody-protein A-agarose complex pellets were collected through centrifugation and washed with RIPA buffer. The bound proteins were separated using SDSPAGE and were then transferred to PVDF membranes for Western blot analyses with anti-mfn2 (Thermo Scientific), anti-Ras (Santa Cruz Biotechnology) or non-specific IgG antibodies (Santa Cruz Biotechnology).

\section{Western blot analysis}

Western blot was performed to determine the expression levels of $m f n 2$, Ras, collagen IV and TFAP2A proteins in GMCs. In brief, cells were pelleted and directly lysed with $2 \%$ SDS lysis buffer, boiled for $10 \mathrm{~min}$ and quantified by NanoDrop 2000. About $10 \mu \mathrm{g}$ of protein was loaded in 4-12\% NuPAGE Bis-Tris gels (Invitrogen), subjected to electrophoresis and transferred to Immobilon-P PVDF Membranes (Millipore). Membranes were blocked with $5 \%$ non-fat milk in Tris-buffered saline containing $0.05 \%$ Tween 20 (Invitrogen) before incubation overnight at $4^{\circ} \mathrm{C}$ with primary antibodies against $m f n 2$ (Thermo Scientific), Ras (Santa Cruz), collagen IV (Santa Cruz) and TFAP2A (ABCAM). Protein bands were visualized using HRP (Bio-Rad), as well as secondary antibodies (Sigma) and SuperSignal West Pico (Thermo Scientific).

\section{Laser confocal microscopy for mitochondrial morphology}

Rat mesangial cells were grown on coverslips inside a Petri dish filled with DMEM culture medium. When cells reached $80 \%$ confluency, the medium was removed from the dish, and a prewarmed $\left(37^{\circ} \mathrm{C}\right)$ staining solution containing diluted MitoTracker Red CMXRos M7512 (Invitrogen) was added to the final working concentration $(300 \mathrm{nM})$ as described previously (Amiott et al. 2008). The mixture was incubated for $30 \mathrm{~min}$ under growth conditions. Afterward, the cells were stained, washed with PBS and fixed with growth medium containing $3 \%$ formaldehyde at $37^{\circ} \mathrm{C}$ for $15 \mathrm{~min}$. The cells were then subjected to permeabilization with $0.2 \%$ Triton $\mathrm{X}-100$ for $10 \mathrm{~min}$. Finally, the coverslips were removed and placed on a dry platform for laser confocal microscopy by using a Zeiss LSM700 system with excitation and emission wavelengths of 579 and $599 \mathrm{nM}$, respectively.

\section{Cellular ROS detection}

ROS production in rat mesangial cells was assessed by flow cytometry (Becton Dickinson, Mountain View, CA, USA) as described previously, with minor modifications (Park et al. 2011). In brief, GMCs from each group under different conditions were washed twice with PBS with 10\% FBS containing $5 \mu \mathrm{mol} / \mathrm{L}$ fluorescent probe CM-H2DCF-DA (Invitrogen). After incubation at routine temperature(RT) for $30 \mathrm{~min}$ in the darkness, cells were washed and resuspended in PBS. DCF intensity was analyzed using flow cytometry with excitation and emission wavelengths of 488 and $530 \mathrm{nM}$, respectively.

\section{Hydrogen peroxide measurement}

Hydrogen peroxide was measured using an Amplex Red Hydrogen Peroxide/Peroxidase Assay Kit (Invitrogen) according to the manufacturer's instructions. In brief, after treating the cells, media supernatants were collected and centrifuged immediately before use. For tissues, the total protein content in each sample was quantified by the Bradford method. A lower hydrogen peroxide concentration range was used for a standard curve to quantify $\mathrm{H}_{2} \mathrm{O}_{2}$ in supernatants. Hydrogen peroxide levels in supernatants were measured by characteristic fluorescence (excitation of $530 \mathrm{~nm}$ and emission of $590 \mathrm{~nm}$ )(Moreira et al. 2015).

\section{Gene knockdown}

GMCs were seeded and transfected at $60 \%$ confluence. Lipofectamine 3000 Reagent (Invitrogen) and $10 \mathrm{nM}$ TFAP2A siRNA (a total of five sequences are listed in Table 1) were diluted in Opti-MEM medium, mixed together and added to cells according to the manufacturer's protocols. After $48 \mathrm{~h}$, cells were used for further experiments.

\section{Quantitative real-time PCR}

GMCs were lysed with TRIzol-LS immediately (Ambion), and total RNA was prepared with RNeasy Plus Mini

Published by Bioscientifica Ltd 
Table 1 siRNA sequences for TFAP2A knockdown.

\begin{tabular}{rrl}
\hline S 5': & CUGGGAGCAUUAACUUUAU UU \\
& mRNA: & ggctgggagcattaactttatta \\
& AS 3': & UU GACCCUCGUAAUUGAAAUA \\
S 5': & CCAGAUCAAACUGUAAUUA UU \\
mRNA: & tccagatcaaactgtaattaag \\
& AS 3': & UU GGUCUAGUUUGACAUUAAU \\
& S 5': & GAGCAGGGUAUCAUUUAGA UU \\
& mRNA: & cagagcagggtatcatttagata \\
& AS 3': & UU CUCGUCCCAUAGUAAAUCU \\
& S 5': & GGUACAACCACCCAUUUGA UU \\
& mRNA: & aaggtacaaccaccatttgaac \\
& AS 3': & UU CCAUGUUGGUGGGUAAACU \\
& S 5': & GUAGGUCAAUCUCCCUACA UU \\
& mRNA: & ctgtaggtcaatctcctacacc \\
& AS 3': & UU CAUCCAGUUAGAGGGAUGU
\end{tabular}

Kit (Qiagen 74134) according to the manufacturer's instructions. cDNA was obtained by reverse transcription by using iScript cDNA Synthesis Kit (Bio-Rad). Afterward, cDNA was amplified with specific primers and detected using a SYBR Green Supermix (Bio-Rad), with the BIO-RAD CFX-96 Real-Time PCR system (Bio-Rad) under the following conditions: $95^{\circ} \mathrm{C}$ for $3 \mathrm{~min}$, followed by 40 cycles of $95^{\circ} \mathrm{C}$ for $10 \mathrm{~s}$ and $55^{\circ} \mathrm{C}$ for $30 \mathrm{~s}$. The relative expression amount of cDNA was calculated using the $2^{-\Delta \Delta \mathrm{Ct}}$ method. Expression levels were normalized with glyceraldehyde-3-phosphate dehydrogenase(GAPDH) mRNA as an internal control. (The primers for collagen IV are listed in Table 2.)

\section{Mfn2 overexpression in vivo}

Male Sprague-Dawley rats (10 weeks old, weighing 200-250g) were purchased from the Experimental Animal Center of Sichuan University. Diabetes was induced with a single intraperitoneal injection of streptozotocin (STZ) (Sigma) at a dose of $65 \mathrm{mg} / \mathrm{kg}$ dissolved in $0.05 \mathrm{M}$ citrate monosodium (ACROS) buffer (Brosius et al. 2009). All animals injected with STZ developed diabetes, as indicated by plasma glucose levels $(13.9 \mathrm{mmol} / \mathrm{L}$ at $48 \mathrm{~h}$ following the injection using a ReliOn Ultima glucose reader (Solartek)) (Lin et al. 2013). The animals were fed under the same condition without insulin therapy, subjected to different treatments, and then killed at the 12th week. Subsequently, kidney tissues were harvested. For determining $\mathrm{M} f \mathrm{f} 2$ overexpression, the animals were first anesthetized with an intramuscular injection of ketamine $(35 \mathrm{mg} / \mathrm{kg})$ and xylazine $(5 \mathrm{mg} / \mathrm{kg})$. After median incision of the abdomen, the abdominal aorta below the renal vessels, inferior vena cava and hepatic portal vessel were clamped. Approximately $15 \mu \mathrm{L}$ of the lentiviral vector-containing solution (either lenti-GFP or lenti-GFP-Mfn2, titer at $1 \times 10^{8}$ tuberculin units $/ \mathrm{mL}$ ) was injected into the renal artery. After blood flow was re-established, the animals were allowed to recover. All animal procedures were approved by the Institutional Animal Care and Use Committee of the West China Hospital of Sichuan University, China.

\section{Renal histology}

The kidney tissues of rats were harvested and placed in $4 \%$ PBS-buffered paraformaldehyde for $24 \mathrm{~h}$ and then embedded in paraffin after killing for each group. The paraffin-embedded tissues were sectioned at 3-5 $\mu \mathrm{m}$ and stained with periodic acid Schiff (PAS). Digital images were obtained from a Leica DM5000B microscope system. PAS staining was used for assessing ECM deposition in glomeruli. The scores of sections were investigated in a blinded manner according to a previous scoring system (range, 0-4: 0, no ECM deposition; 4, ECM deposition in all sections of the glomeruli) (Tak et al. 2013).

\section{Isolation of glomeruli}

Glomeruli were isolated from the kidneys of rats by sequential sieving, as described previously (Holdsworth et al. 1978). In brief, after the rats were killed, kidneys were removed and de-capsulated. The renal cortical tissues were cut into $1 \mathrm{~mm}^{3}$ pieces, ground gently on $250 \mu$ stainless mesh with a syringe handle and rinsed with $1 \times$ PBS. The filtered material was sequentially passed through 100 and $70 \mu$ nylon meshes (Biologix, Jinan, China). The glomeruli-enriched fraction was retained on top of the $70 \mu$ mesh and subjected to further experiments.

\section{Chromatin immunoprecipitation (ChIP)}

For each ChIP experiment, approximately $1 \times 10^{6}$ cells were fixed with $1 \%$ formaldehyde at RT for $5 \mathrm{~min}$, lysed,

Table 2 Primers for collagen IV in q-PCR.

\begin{tabular}{|c|c|c|}
\hline Primer name & Forward 5'-3' & Reverse 5'-3' \\
\hline Collagen IV-a1 cDNA & GAAAGGAGACCAGGGAGA & TCCTCGGGAACCTTTATC \\
\hline GAPDH & GTTACCAGGGCTGCCTTCTC & GATGGTGATGGGTTTCCCGT \\
\hline
\end{tabular}


prepared for sonication with TruChIP Chromatin Shearing Reagent kit (Thermo Scientific, Covaris, 520127) and sonicated using Covaris E220 system to shear chromatin DNA. TFAP2A and H3K27 acetylationbound chromatin fragments were enriched by immunoprecipitation with rabbit pAbs (Abcam) raised against the two immunogens. The ChIP reactions were carried out with a ChIP-IT high-sensitivity kit (Active Motif, 53040). After reversal of cross-linking, enriched DNA fragments were purified using a MinElute PCR purification kit (Qiagen, 28004) (Fan et al. 2012). Enrichments for specific DNA binding or histone modifications were quantified by qPCR.

\section{Quantitative real-time PCR for enriched DNA segments after ChIP reaction (ChIP-qPCR)}

TFAP2A binding and H3K27 acetylation enrichments were assessed by ChIP-qPCR in GMC. After the ChIP step, enriched DNA segments from matched IP sample and input control sample (without ChIP reactions) were subjected to quantitative real-time PCR (qPCR) analysis using SYBR Green Supermix (Bio-Rad) and CFX96 Realtime PCR system (Bio-Rad). qPCR positive primers were designed to cover the core consensus of three motifs (All primers are listed in Table 3). qPCR negative primers were referenced to the known negative regions. Enrichment scores were computed as $2^{-\Delta \Delta \mathrm{Ct}}$ using the following formula:

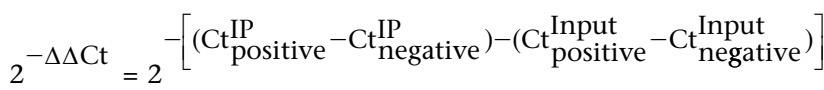

(IP: DNA after ChIP reaction; Input: DNA without ChIP reaction; Positive: positive region primer pair; Negative: negative primer pair) (All primers for ChIPqPCR are listed in Table 3.)

\section{Statistical analysis}

All data are expressed as the mean \pm S.E.m. The comparison between groups was performed using one-way ANOVA. $P<0.05$ was considered statistically significant. SPSS statistical software for Mac (version 20.0) was used for analysis.

\section{Results}

\section{Effects of AGE on expression of Mfn2 and production of ROS}

In a previous study, we found that the decreased expression of $M f n 2$ induced by high glucose is combined with mitochondrial dysfunction (Tang et al. 2012). In this study, we detected the expression of $M f n 2$ protein in GMCs stimulated by AGE at 0, 24, 36, 48 and $60 \mathrm{~h}$. The results indicated that $M f n 2$ expression did not change over time in the control group (Fig. 1A, line 1). By contrast, with AGE stimulation, Mfn2 expression declined significantly at $36 \mathrm{~h}$, dropped to the lowest level at $48 \mathrm{~h}$ and showed no further decline at $60 \mathrm{~h}$ (Fig. 1A, line 3). Inversely, AGE increased cellular ROS production over time, peaking at $48 \mathrm{~h}$ (Fig. 1B), which was $1.77 \pm 0.09$-fold higher than that at $0 \mathrm{~h}(P<0.05)$. Furthermore, changes in the hydrogen peroxide $\left(\mathrm{H}_{2} \mathrm{O}_{2}\right)$

Table 3 Primers for ChIP q-PCR.

\section{Primer name}

Region1-core

Region $1-(-400 \mathrm{bp})$

Region 1-(-200 bp)

Region1-(+200 bp)

Region 1-(+400 bp)

Region2-core

Region2-(-400 bp)

Region2-(-200 bp)

Region2-(+200 bp)

Region2-(+400 bp)

Region3-core

Region3-(-400 bp)

Region3-(-200 bp)

Region3-(+200 bp)

Region3-(+400 bp)

TFAP2A negative

H3K27ac negative
Forward 5'-3

GGCAGGAAGGAGACAT

CAGATAGGTGAGGGAAAG

TAAACAGCACTACCTATGAG

CTCAAGATTTGGGACAGA

TTGGCAGCAAAAGGTG

TGCATTGCACAGGACA

ATAGGAGAAGGAGGGAGG

GACCAGAGGCTGAGAAC

AGACTCTTCTCCGTAGGC

CAAGGGGCTCGTGGAA

AGGGGCTCGTGGAATC

GCAGCGCCAACAAATT

CGCTGCGAAGGTGAGT

ATCGCACATCCATTACG

CCACTCAGAGTCCCACA

AGAGGAAGGCTACTGATT

AGCTGCTGGTTTTCTTCA

\begin{tabular}{l} 
Reverse $\mathbf{5}^{\prime} \mathbf{-} \mathbf{3}^{\prime}$ \\
\hline CCTGCTCTGCTGGGAT \\
CCAGTGCCTAGACAGAAC \\
TAAATGTAGCCAATCCTT \\
GAAACTCAGCGGTGGT \\
CGAGTGCGTGTAGGTGT \\
GGAAGACAGGCTCACC \\
AGGGGACTTTGAATGTATCT \\
GCTGCGATGATAATGC \\
GCTGCATGGGCTGAAC \\
CGCACCCAGCTCTTCC \\
GGCTCGGGACTTGGTG \\
GCGAGGTGCTCAGTCT \\
CGCTGGACAAAGAGGC \\
GCTTCAGTCCCTTCCTAA \\
CTCCAAACAACCAGAAAC \\
CCACAAAAGTTCCACCA \\
AGAATGCTTACATAGTCCC
\end{tabular}

Reverse 5'-3'

TAAATGTAGCCAATCCTT

GAAACTCAGCGGTGGT

CGAGTGCGTGTAGGTGT

GGAAGACAGGCTCACC GCTGCGATGATAATGC GCTGCATGGGCTGAAC CGCACCCAGCTCTTCC GGCTCGGGACTTGGTG GCGAGGTGCTCAGTCT CGCTGGACAAAGAGGC GCTTCAGTCCCTTCCTAA CCACAAAAGTTCCACCA AGAATGCTTACATAGTCCC http://jme.endocrinology-journals.org DOI: 10.1530/JME-16-0031
๑) 2016 Society for Endocrinology Printed in Great Britain 
concentration over time under AGE stimulation showed a similar trend as ROS (Fig. 1C). These results indicate that AGE reduces $M f n 2$ expression and increases ROS production simultaneously, and further suggest that Mfn2 expression changes are closely correlated with ROS production in DN. Based on these trends, $48 \mathrm{~h}$ of AGE stimulation is the optimal experimental condition for conducting further experiments in vitro. Beyond that, we found that pre-treatment with $\mathrm{N}$-acetylcysteine (NAC) could also effectively reduce ROS or $\mathrm{H}_{2} \mathrm{O}_{2}$ production induced by AGE (Fig. 1D). In addition, blocking ROS with $\mathrm{N}$-acetylcysteine (NAC) had a slight reverse effect on the decreased $m f n 2$ expression under AGE stimulation (Fig. 1E). For these reasons, we chose NAC as the ROS blocker. Taken together, our results suggest that decreased expression of $M f n 2$ after AGE stimulation may serve as a distinct feature of dysfunctional mitochondria associated with ROS overproduction.

\section{Interaction between Mfn2 and Ras in vitro}

To further elucidate the unique direct effect of $M f n 2$ on mitochondria, we deleted the Ras-binding site sequences (77th to 91st amino acids) while conserving the domain
A

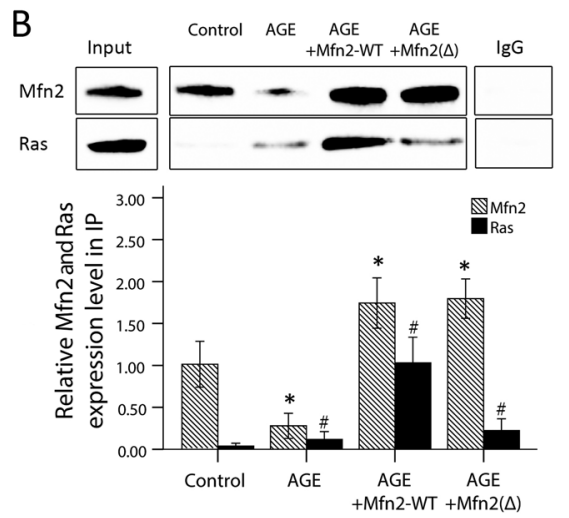

D

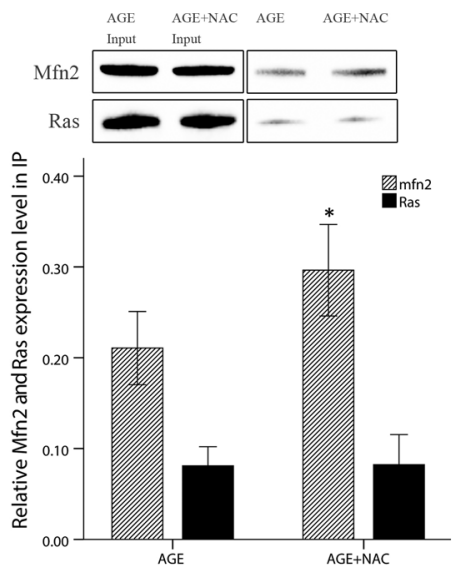

E

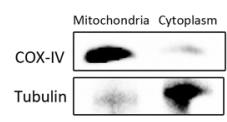

$\mathrm{F}$

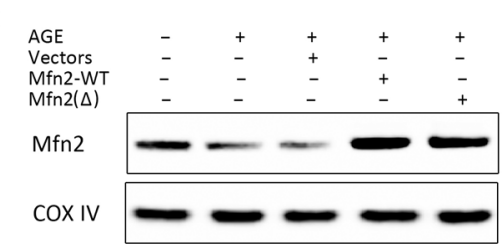

C

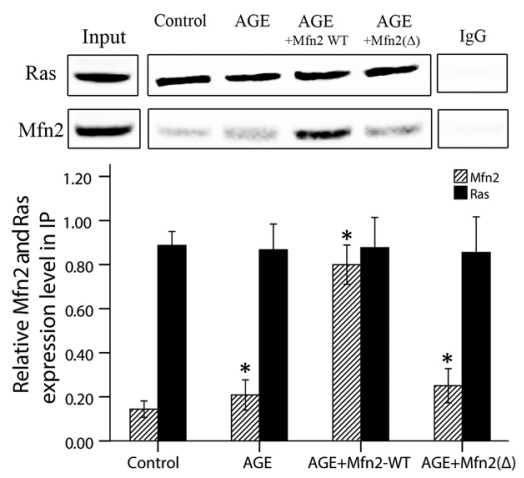

Figure 2

The effects of overexpression of $\mathrm{Mfn} 2-\operatorname{Ras}(\Delta)$ on mfn2-Ras binding and $\mathrm{mfn} 2$ mitochondrial distribution. (A) The sequencing image for wild-type mfn2 or Mfn2-Ras( $\Delta$ ) mRNA. The upper part: for wild-type mfn2, the Ras-binding sequences were marked with grey background. The white triangle indicates the region of Ras-binding sequences; the lower part: for $\operatorname{Mfn} 2-\operatorname{Ras}(\Delta)$, the black triangle indicates the start site from which the Ras-binding domain has been deleted. (B) Overexpression of Mfn2-Ras( $\Delta$ ) did not enhance the Mfn2-Ras binding after AGE stimulation by co-immunoprecipitation. GMCs were exposed to control BSA or AGE at the concentration of $40 \mu \mathrm{M}$ for $48 \mathrm{~h}$. The top lane represents IP with Mfn2 and blot for Mfn2; the bottom lane IP with Mfn2 and blot for Ras. Input-positive control: the expression of Ras or $\mathrm{mfn} 2$ without co-immunoprecipitation; IgGnegative control, co-immunoprecipitation for Ras and $\mathrm{mfn} 2$ using non-specific Ig $\mathrm{G}$ antibody. $* P<0.05$, IP with Mfn2 and blot for Mfn2 vs control group; ${ }^{*} P<0.05$, IP with Mfn2 and blot for Ras vs control group. (C) Mfn2 and RAS may co-immunoprecipitate with each other after AGE stimulation. GMCs were exposed to control BSA or AGE at the concentration of $40 \mu \mathrm{M}$ for $48 \mathrm{~h}$. The top lane represents IP with Ras and blot for Ras; the bottom lane IP with Ras and blot for Mfn2. Input-positive control: the expression of Ras or $\mathrm{mfn} 2$ without co-immunoprecipitation; IgG-negative control, co-immunoprecipitation for Ras and $\mathrm{mfn} 2$ using non-specific lgG antibody. $* P<0.05$, IP with Ras and blot for Mfn2 vs control group. (D) ROS blocking reversed the decrease of $\mathrm{mfn} 2$ induced by AGE to a small extent, but had no effect on Mfn2-Ras binding. The samples were pretreated with NAC $(5 \mathrm{mM})$ for $2 \mathrm{~h}$ before the addition of AGE in AGE + NAC group. ${ }^{*} P<0.05$, IP with Mfn2 and blot for Mfn2 vs AGE group. (E) The purity of mitochondrial fractions. COX-IV, a mitochondrial protein; tubulin, a cytoplasmic protein. (F) The expression of $\mathrm{mfn} 2$ protein by Western blotting on isolated mitochondria. COX-IV was used as an internal control. AGE stimulation is at a final concentration of $40 \mu \mathrm{M}$ for $48 \mathrm{~h} .{ }^{*} P<0.05$ vs control group. Data are mean \pm s.E.M. of three separate experiments. 
mediating mitochondrial function. Sequences of both wild-type and $M f n 2-\operatorname{Ras}(\Delta)$ mRNA are shown in Fig. 2A. Co-immunoprecipitation was used to detect the interaction between Ras and $M f n 2$ proteins. After $48 \mathrm{~h}$ of stimulation with AGE, we found that either $M f n 2$ expression or Ras-Mfn2 binding increased (Fig. 2B, lane 2). Overexpression of wild-type $M f n 2$ significantly increased the amount of $M f n 2$ protein and Ras-Mfn2 binding at the same time (Fig. 2B, lane 3). Mfn2-Ras( $\Delta$ ) overexpression also notably facilitated the expression of $M f n 2$ protein but did not increase Ras-Mfn 2 binding (Fig. 2B, lane 4), demonstrating that $M f n 2-\operatorname{Ras}(\Delta)$ cannot bind to Ras and only has the ability to interact with mitochondria.

In reciprocal immunoprecipitation experiments with RAS and immunoblot for Mfn2 (Fig. 2C), AGE did not change Ras protein expression but slightly increased the amount of Ras-Mfn2 binding (Fig. 2C, lane 2).
Moreover, wild-type $M f n 2$ overexpression significantly increased the amount of Ras-Mfn 2 binding but not Mfn2-Ras( $\Delta$ ) overexpression (Fig. 2C, lane 3,4). These results suggest that only $M f n 2$ with the Ras-binding domain and RAS can co-immunoprecipitate with each other. $M f n 2-\operatorname{Ras}(\Delta)$ eliminates the potential for a Ras signaling mechanism, thereby providing us with a tool to examine the unique mitochondria-protective function of this protein.

In our study, NAC treatment of GMC had a modest effect on $M f n 2$ expression, and the decrease in $M f n 2$ with AGE treatment of GMC was only modestly reversed by the ROS blocker NAC (Fig. 2D, first line, right lane). However, it had barely any effect on Mfn2-Ras binding (Fig. 2D, second line, right lane). These results suggest that the dysfunctional mitochondria overproduce ROS and that ROS has adverse effects on mitochondria for facilitating mitochondrial damage.

A
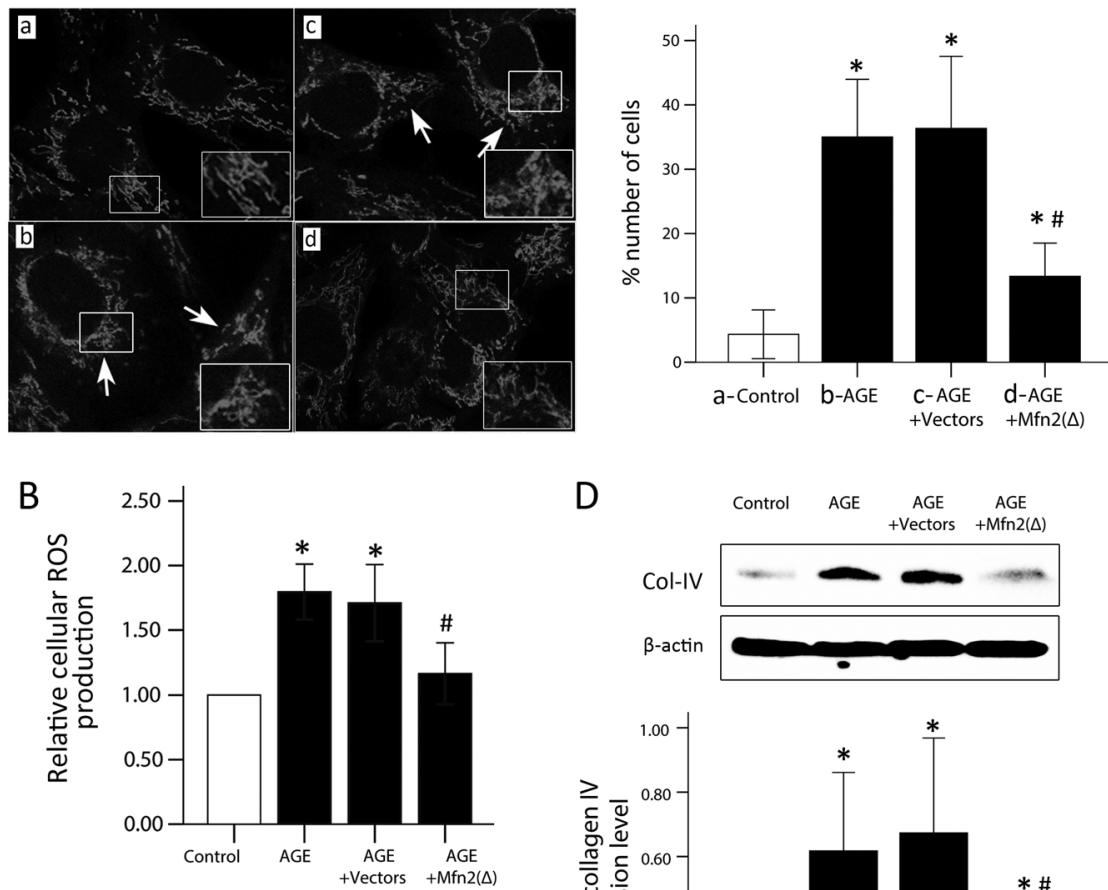

\section{Figure 3}

Overexpression of $\mathrm{Mfn} 2-\operatorname{Ras}(\Delta)$ ameliorated the mitochondrial morphology and function, thus reducing collagen IV synthesis. (A) The mitochondrial morphology changes in different groups by laser scanning confocal microscopy. The white arrows indicate the fragment mitochondria. Views with high magnification were shown at the low right box. The right part is quantitation result for $\%$ number of cells with fragmental mitochondria, $* P<0.05$ vs control group; ${ }^{P}<<0.05$ vs AGE group. (B) The relative level of ROS after Mfn2-Ras $(\Delta)$ transfection. ${ }^{*} P<0.05$ vs control group; ${ }^{\#} P<0.05$ vs $A G E$ group. (C) The hydrogen peroxide concentration changed after Mfn2-Ras $(\Delta)$ transfection. ${ }^{*} P<0.05$ vs control group; $\# P<0.05$ vs AGE group. (D) The expression of collagen IV in rat mesangial cells by Western blotting. $\beta$-actin was used as an internal control. ${ }^{*} P<0.05$ vs control group; ${ }^{P} P<0.05$ vs AGE group. Data are mean \pm s.E.M. of three separate experiments.

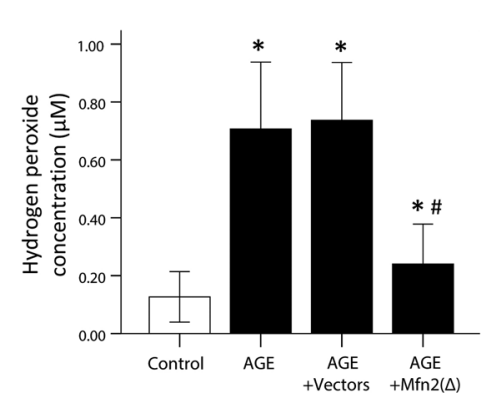




\section{Effects of $M f n 2-\operatorname{Ras}(\Delta)$ overexpression on AGE-induced mitochondrial dysfunction and collagen IV synthesis}

Optimal expression of $M f n 2$ in mitochondria aids in maintaining normal mitochondrial morphology and function (Baumann 2010, Chen et al. 2010a). In this study, either total $m f n 2$ or mitochondrial $m f n 2$ expression decreased after AGE stimulation. By contrast, overexpression of $M f n 2$ increased the amount of $M f n 2$ on mitochondria even with AGE stimulation, indicating that Mfn2 mitochondrial distribution also changed after transfection (Fig. 2F). Furthermore, mitochondrial morphology was examined using MitoTracker labeling and confocal microscopy. The results revealed prominent fragments of mitochondria in the AGE group. Fragmented mitochondria were found to contain small spheres or short rods with AGE stimulation, whereas normal mitochondria were found to shape an elongated network under normal conditions (Fig. 3A). Mitochondrial fragments are related to mitochondrial dysfunction (Detmer \& Chan 2007). This mitochondrial morphological change was accompanied by increase in ROS, up to $1.79 \pm 0.09$-fold compared with that of the control group (Fig. 3B). Beyond this point, ROS overproduction may lead to further damage to cells. Consistent with previous reports (Li et al. 2014, Peng et al. 2015, Yu et al. 2006), Mfn2-Ras( $\Delta$ ) overexpression dramatically reversed all of the previously mentioned adverse indicators of mitochondrial health under AGE stimulation, including the increased $M f n 2$ mitochondrial distribution, elongated mitochondrial morphology and decreased ROS and hydrogen peroxide production, which was $1.17 \pm 0.11$-fold compared with that in the control group and significantly different from that in the AGE group $(P<0.05)$ (Figs $2 \mathrm{~F}$ and $3 \mathrm{~A}, \mathrm{~B}$ and $\mathrm{C}$ ). These results collectively demonstrate that $M f n 2-\operatorname{Ras}(\Delta)$ overexpression rescued AGE-induced mitochondrial dysfunction partially by, but not limited to, alleviating mitochondrial fragmentation. Eventually, expression of collagen IV, a major component of ECM, increased with ROS overexpression under AGE stimulation. The involvement of ROS in increase in collagen IV has previously been reported in numerous studies (Taye et al. 2013, Papadimitriou et al. 2014, Yan et al. 2015). We also found that $M f n 2-\operatorname{Ras}(\Delta)$ overexpression mitigated ROS overproduction along with decreased collagen IV synthesis (Fig. 3D). Our results suggest that $M$ fn2 performs a protective function in DN through the mitochondria-ROS pathway.

\section{Motif search for proximal regions near the transcriptional start site (TSS) of collagen IV}

A computer search of the DNA sequence from chr16:82985857 to chr16:82989857 covering 2000bp upstream and downstream of collagen IV TSS was performed to further investigate how the mitochondrial dysfunction-ROS pathway affects collagen IV expression. First, the sequence was calculated using FIMO software, a motif search tool (Grant et al. 2011), with the command line: 'fimo --oc . --verbosity 1 --thresh 1.0E-4 All-motif. meme sequences.fa.' The results revealed several consensus sequences for binding of the transcription factor TFAP2A (Fig. 4A) in this area. Second, accurate positions of TFAP2A motifs were calculated using the MAST (version 4.10.2) software (Bailey \& Gribskov 1998) with the command line: 'mast motifs.meme sequences.fa -oc . -nostatus -remcorr -minseqs 1 -ev 10.0.'. Results indicate that these sequences lie in the upstream -1497 to $-1474 \mathrm{bp}$ and -625 to $-602 \mathrm{bp}$ and the downstream +288 to $+309 \mathrm{bp}$ of TSS. A high degree of similarity was observed between each identified sequence and TFAP2A $(P<1.0 \mathrm{E}-4)$ (Fig. 4B).

\section{Relationship between TFAP2A and collagen IV}

On account of the interesting results of our bioinformatic analysis, we conducted additional experiments to probe the relationship between TFAP2A and collagen IV. First, we successfully knocked down TFAP2A expression using siRNA. Relative expression of the TFAP2A protein significantly decreased upon siRNA administration to $26.04 \pm 3.89 \%$ of that in the control group without siRNA $(P<0.05)$ (Fig. 4C). Second, by Western blotting, the expression level of TFAP2A after AGE stimulation was found to have no significant difference compared with that in the control group (Fig. 4D). These results indicate that AGE stimulation did not change the expression level of TFAP2A protein in this study. Third, the effect of TFAP2A knockdown on the expression of collagen IV mRNA was investigated by qPCR. Our results show that inhibition of TFAP2A significantly decreased the relative level of collagen IV mRNA from $3.31 \pm 0.21$ to $1.49 \pm 0.12$-fold higher compared with that in the control group $(P<0.05)$ (Fig. $4 \mathrm{E})$. These results indicate that TFAP2A is involved in the regulation of collagen IV expression at the transcriptional level. Furthermore, we found that changes in the level of collagen IV protein were consistent with changes in mRNA levels upon TFAP2A inhibition (Fig. 4F). Taken together, our results

Published by Bioscientifica Ltd. 
A

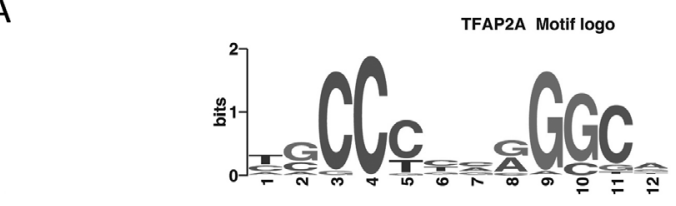

B

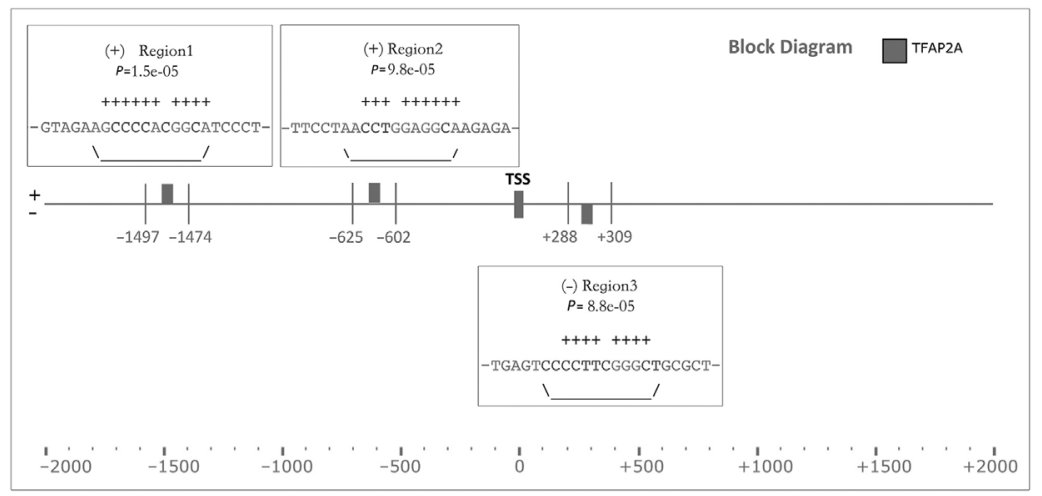

C
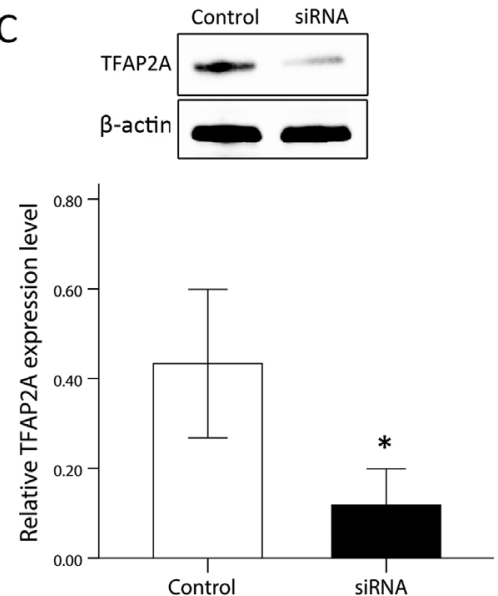

$\mathrm{E}$

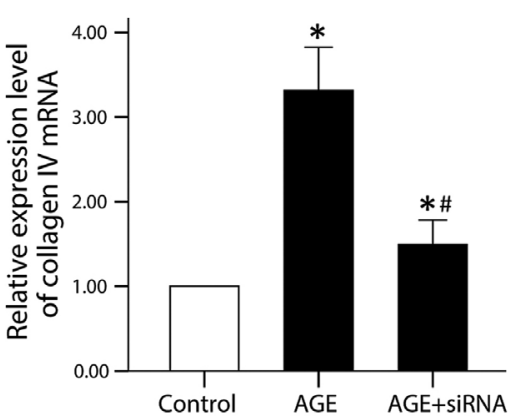

D
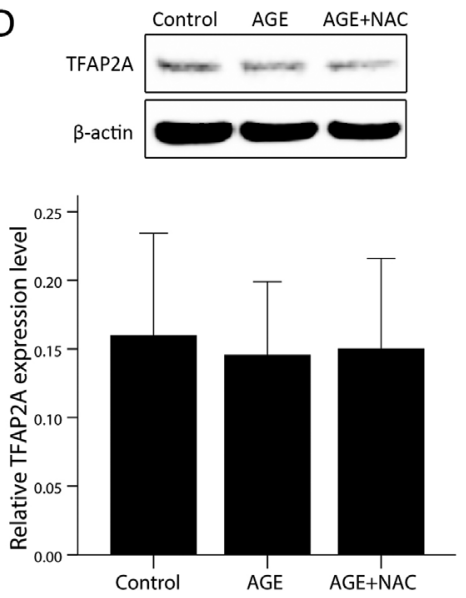

$\mathrm{F}$
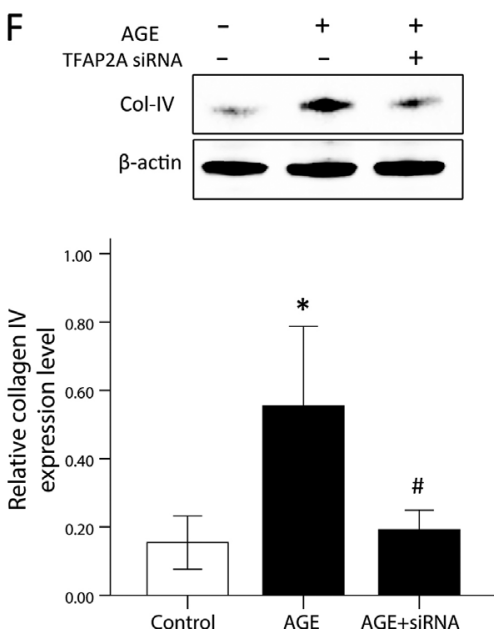

\section{Figure 4}

Motif search for the proximal regions near the TSS of collagen IV and validation for matched transcript factor. (A) The logo of transcript factor TFAP2A. The logo was drawn using meme software according to the occurrence rate for each base of TFAP2A motif. (B) The block diagram of three motifs for TFAP2A binding near the TSS of collagen IV. In each block, the ' $(+)^{\prime}$ indicates the motif locates at forward chain, whereas ' $(-)$ ' at reverse chain; $P$ value is the possibility which means the core sequence is dissimilar to that of the given motif matrix. The ' + ' above each base sequence means it has high similarity to that of the given motif; the core sequence of each motif was marked with colors; the grey block indicates the sequence of TFAP2A; TSS is transcriptional start site; the number ' 0 ' on the scale at the bottom means the location of TSS, upstream locations are indicated in negative numbers, whereas downstream in positive numbers. (C) Western blotting result for knocking down TFAP2A in rat GMCs using siRNA technology. $\beta$-actin was used as an internal control. ${ }^{*} P<0.05$ vs control group. (D) AGE stimulation did not change the expression of TFAP2A protein. $\beta$-actin was used as internal control. (E) Knocking down of TFAP2A has effect on the mRNA expression of collagen IV under AGE stimulation. ${ }^{*} P<0.05$ vs control group; $\# P<0.05$ vs AGE group. (F) Knocking down of TFAP2A has final effect on the expression of collagen IV protein. $\beta$-actin was used as an internal control. ${ }^{*} P<0.05$ vs control group; ${ }^{\#} P<0.05$ vs AGE group. Data are mean \pm S.E.M. of three separate experiments. 
A

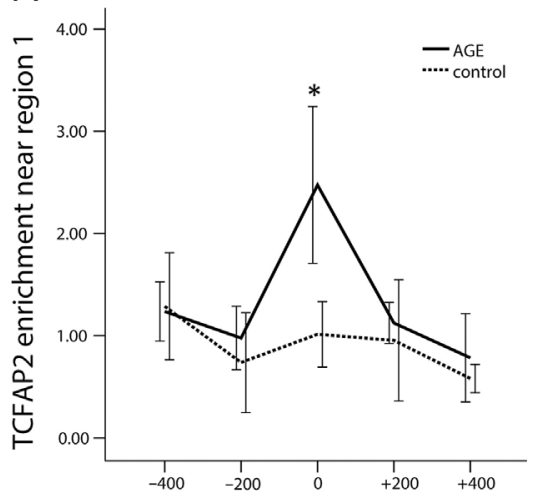

C

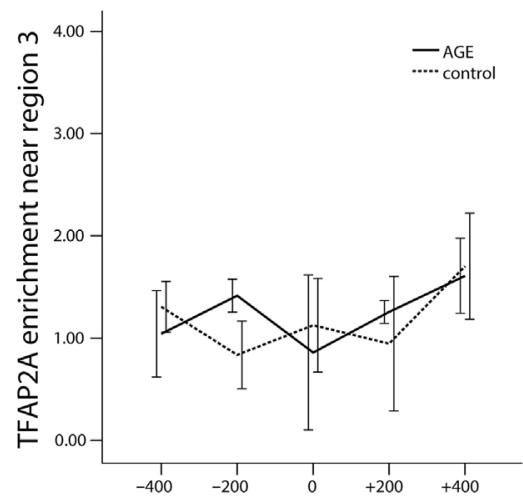

$E$

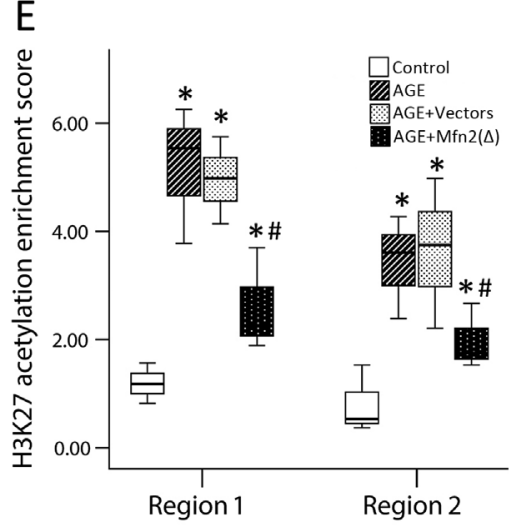

B

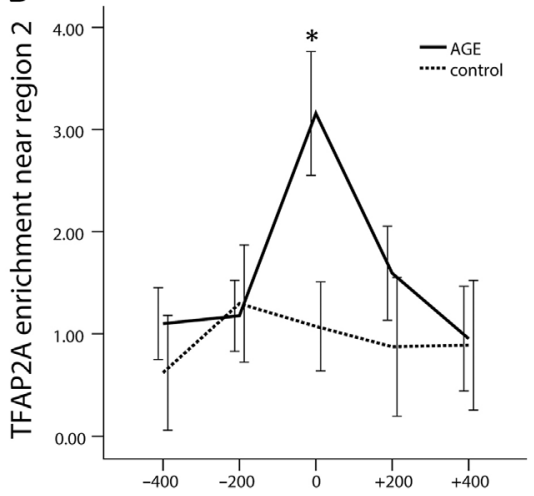

D

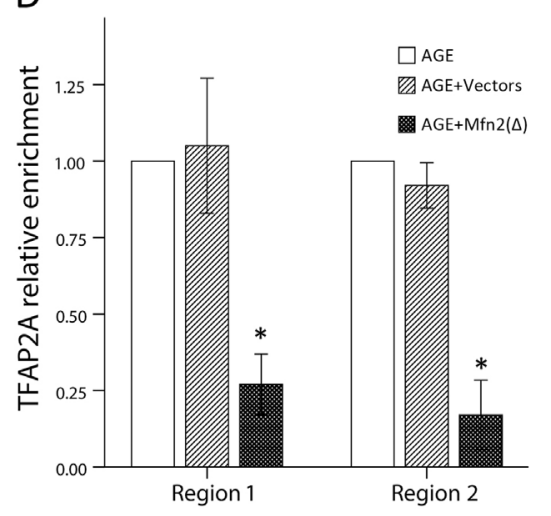

$\mathrm{F}$

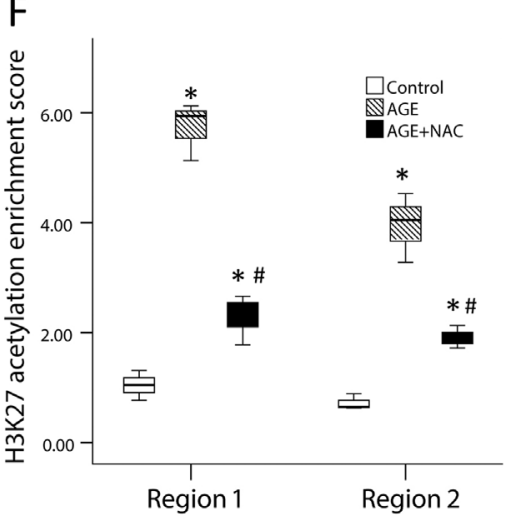

Figure 5

ChIP-qPCR results for TFAP2A DNA binding and the effects of Mfn2-Ras $(\triangle)$ overexpression on H3K27 acetylation. (A) TFAP2A enrichments at Region 1 and near regions within $400 \mathrm{bp}$ distance with or without AGE stimulation. ${ }^{*} P<0.05$ vs the same region without AGE stimulation. (B) TFAP2A enrichments at region 2 and near regions within 400 bp distance with or without AGE stimulation. ${ }^{*} P<0.05$ vs the same region without AGE stimulation. (C) TFAP2A enrichments at Region 3 and near regions within $400 \mathrm{bp}$ distance with or without AGE stimulation. (D) Mfn2-Ras( $\Delta$ ) overexpression has an effect on TFAP2A enrichment at both Regions 1 and 2 . ${ }^{*} P<0.05$ vs vectors transfection under AGE stimulation. (E) The changes of H3K27 acetylation in Regions 1 and 2 after AGE stimulation with or without $\operatorname{Mfn} 2-\operatorname{Ras}(\Delta)$ transfection. ${ }^{*} P<0.05$ vs control group; ${ }^{P} P<0.05$ vs AGE group. (F) ROS blocking has effects on H3K27 acetylation in both Regions 1 and 2. ${ }^{*} P<0.05$ vs control group; ${ }^{*} P<0.05$ vs AGE group. Data are mean \pm S.E.M. of three separate experiments. indicate that TFAP2A has a positive regulatory function in the synthesis of collagen IV in DN.

\section{Effects of AGE on TFAP2A DNA binding}

Subsequently, we performed TF-ChIP experiments to test the DNA binding of TFAP2A at the corresponding motif regions with or without AGE stimulation. As shown in Fig. 5A, no TFAP2A enrichment peaks were found at the motif center ( $0 \mathrm{bp}$ ), as well as $-400,-200,+200$ and +400 bp from the motif center of Region 1 without AGE stimulation. On the contrary, with AGE stimulation, the signal of TFAP2A enrichment was found at the motif center (Obp), which was significantly stronger than that without AGE stimulation at the same site $(P<0.05)$. Similar results were also found in Region 2, as shown in Fig. 5B. The results indicate that AGE stimulation observably increased the DNA binding of TFAP2A at the motif centers of Regions 1 and 2, consequently facilitating the regulatory function of TFAP2A. However, unlike Regions 1 and 2, TFAP2A-binding enrichment signals have not been detected at Region 3 (Fig. 5C), suggesting that not 
all calculated motifs could bind with the corresponding TFs under certain conditions. Hence, Region 3 may not be involved in this pathological process and may instead participate in another unknown mechanism.

\section{Effect of $M f n 2-\operatorname{Ras}(\Delta)$ overexpression on TFAP2A DNA binding}

As described previously, $M f n 2-\operatorname{Ras}(\Delta)$ overexpression mitigates the synthesis of collagen IV in DN. Therefore, we conducted another TF-ChIP experiment to detect the effect of $M f n 2-\operatorname{Ras}(\Delta)$ overexpression on TFAP2A DNA binding at Regions 1 and 2. Our results reveal that TFAP2A relative enrichment at Region 1 was significantly reduced by $M f n 2-\operatorname{Ras}(\Delta)$ overexpression to $0.27 \pm 0.04$-fold of that in the AGE group $(P<0.05)$. Mfn2-Ras $(\Delta)$ overexpression also decreased TFAP2A enrichment at Region 2 to
$0.17 \pm 0.05$-fold of that in the AGE group $(P<0.05)$ (Fig. 5D). Correspondingly, vector transfection did not manifest these effects. Taken together, these consequences demonstrate that $M f n 2-\operatorname{Ras}(\Delta)$ overexpression attenuates abnormal TFAP2A DNA binding, contributing to inhibition of the mechanism driving collagen IV gene expression through the mitochondria-ROS-TF pathway in DN.

\section{Epigenetic changes in TFAP2A-binding sites}

Furthermore, considering that the transcription factor TFAP2A can bind at Regions 1 and 2 near the collagen IV TSS and enhance gene expression, whether these two regions possess epigenetic modifications remains unclear. Our results suggest H3K27 acetylation of both Regions 1 and 2 at low levels under normal conditions.
A

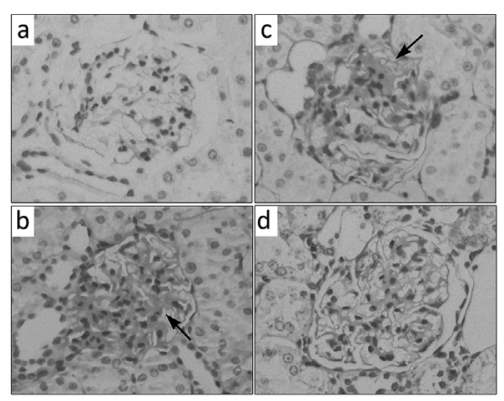

C

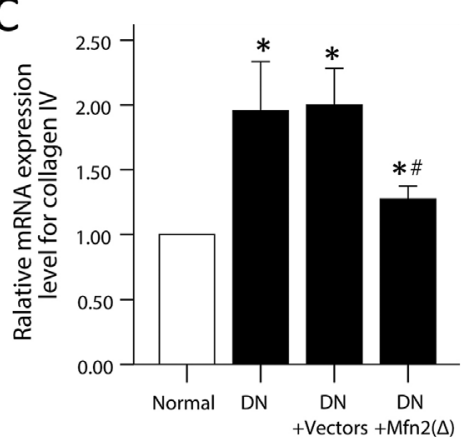

$\mathrm{E}$

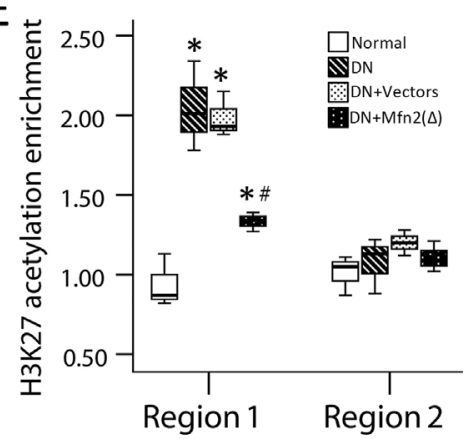

B

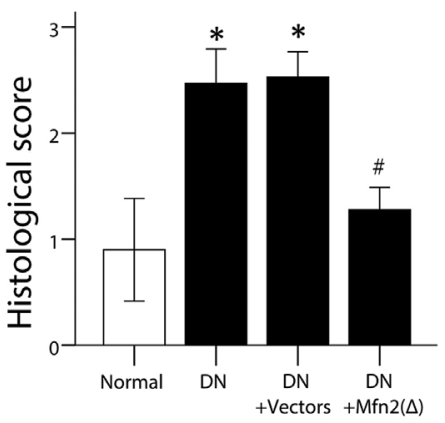

D

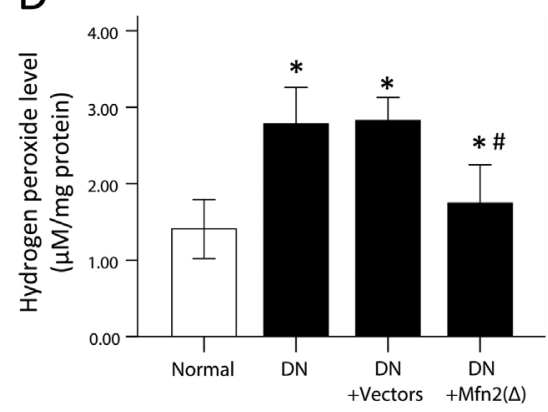

$\mathrm{F}$

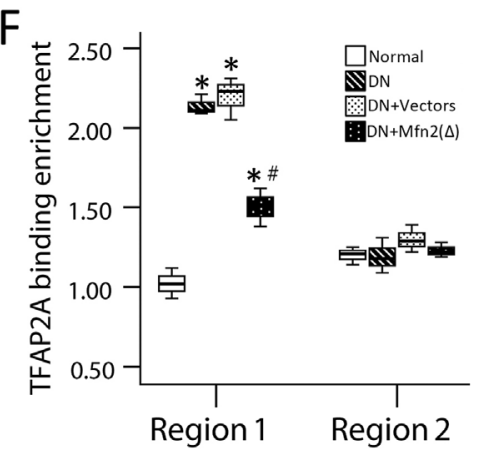

Figure 6

Extracellular matrix deposition, TF binding and $\mathrm{H} 3 \mathrm{~K} 27$ acetylation in vivo. (A) Extracellular matrix deposition as determined by PAS staining. Black arrows indicate the ECM deposition. In Fig. 6(Aa) Normal rats as control; (Ab) diabetic rats without Mfn2-Ras $(\Delta)$ transfection; (Ac) diabetic rats with vectors transfection in vivo; (Ad) diabetic rats with $\operatorname{Mfn} 2-\operatorname{Ras}(\Delta)$ transfection in vivo.

(B) Histological scores of PAS staining sections. The scores were assessed at least in ten glomeruli in sections of three different mice. ${ }^{*} P<0.05$ vs normal group; ${ }^{\#} P<0.05$ vs DN group. (C) Relative mRNA expression level for collagen IV in isolated glomeruli of rats as determined by $q-P C R$. * $P<0.05$ vs normal group; $\#<0.05$ vs $D N$ group. (D) The hydrogen peroxide concentration changed after $\operatorname{Mfn} 2-\operatorname{Ras}(\Delta)$ transfection in vivo. * $P<0.05$ vs normal group; $P<0.05$ vs DN group. (E) The changes of H3K27 acetylation enrichment in Regions 1 and 2 in isolated glomeruli of rats. * $P<0.05$ vs normal group; $P<0.05$ vs DN group. (F) The changes of TFAP2A binding enrichment in Regions 1 and 2 in isolated glomeruli of rats. * $P<0.05$ vs normal group; $\#<0.05$ vs $D N$ group. Quantitative data are reported as the mean \pm S.D. (Six animals were used for 6 independent experiments in each group). 
On the contrary, the levels of H3K27 acetylation of Regions 1 and 2 are notably increased under AGE stimulation and can be mitigated by Mfn2$\operatorname{Ras}(\Delta)$ overexpression. In comparison, vector transfection showed no effect (Fig. 5E). To investigate the effect of ROS on H3K27 acetylation, we used ChIP-qPCR for determining the H3K27 acetylation level. In our study, we found that AGE significantly increased H3K27 acetylation level in both Regions 1 and 2. Interestingly, ROS blocking with NAC significantly decreased H3K27 acetylation in the two regions (Fig. 5F). The results indicate that ROS blocking has negative effect on the H3K27 acetylation level in Regions 1 and 2 after AGE stimulation. Mfn2$\operatorname{Ras}(\Delta)$ overexpression, like ROS blocking, can mitigate the H3K27 acetylation level in Regions 1 and 2 after AGE stimulation. The protective role of $M f n 2-\operatorname{Ras}(\Delta)$ overexpression may be responsible for its effects on decreasing ROS production (Fig. 3B). These novel results are consistent with changes in TFAP2A binding under different conditions. Thus, H3K27 acetylation at the two regions may facilitate subsequent TFAP2A binding.

\section{In vivo investigation of the effects of $M f n 2-\operatorname{Ras}(\Delta)$ overexpression on epigenetic changes at TFAP2A-binding sites}

To further validate the epigenetic changes at TFAP2Abinding regions in vivo, we established STZ-induced diabetic animal models and overexpressed $M f n 2-\operatorname{Ras}(\Delta)$ in their kidneys through targeted transfection. Consistent with our previous results, Mfn2-Ras( $\Delta)$ overexpression in vivo notably attenuated glomerular mesangial lesions (including ECM deposition and mesangial area expansion) and significantly decreased mesangial pathological scores (Fig. 6A and B). To further verify whether Mfn2$\operatorname{Ras}(\Delta)$ overexpression affected collagen IV expression at the transcriptional level, we detected glomerular collagen IV gene expression by qPCR. In accordance with the results in vitro, an evident increase in collagen IV gene expression in diabetic animal can be reversed by $M f n 2-\operatorname{Ras}(\Delta)$ overexpression at the transcriptional level (Fig. 6C). In DN models, hydrogen peroxide production increased significantly, whereas in the $M f n 2-\operatorname{Ras}(\Delta)$ overexpression group, hydrogen peroxide production was significantly reversed (Fig. 6D). The results indicate that $M f n 2-\operatorname{Ras}(\Delta)$ overexpression can mitigate hydrogen peroxide production in DN animals. Interestingly, H3K27 acetylation changes were detected at Region 1 with notable increase in the glomerulus of diabetic animals. However, H3K27 acetylation signals were fairly weak at Region 2 both in diabetic and normal animals (Fig. 6E). Consequently, TFAP2A binding changed similarly at the two regions (Fig. 6F). Moreover, Mfn2-Ras $(\Delta)$ overexpression in vivo significantly decreased both H3K27 acetylation and TFAP2A binding at Region 1. These results suggest that expression of collagen IV in DN in vivo depends on a considerably more complex regulatory mechanism than that in vitro.

\section{Discussion}

AGEs, as well as hyperglycemia, are known to be critical factors in the progression of DN (Brownlee et al. 1988, Soulis-Liparota et al. 1995). Here, we found that AGE significantly increased ROS production in GMCs, which is consistent with previous reports. In vivo experiments strongly demonstrated that infusion of AGEs into mice leads to increased cytosolic ROS, followed by increased mitochondrial permeability and deficiency of the mitochondrial respiratory complex I. By contrast, use of AGE cross-link breaker agent reduces mitochondrial superoxide generation (Coughlan et al. 2009). Moreover, similar results were found in cultured renal mesangial cells (Ide et al. 2010) and interstitial fibroblasts (Chen et al. 2010b). In our study, we also revealed that decreased expression of $M f n 2$ over time was accompanied by ROS overproduction under AGE stimulation. The downregulation of $M f n 2$ has been proven previously in cultured renal tubular cells with high glucose (Zhan et al. 2014) and in DN rat models (Tang et al. 2012). Interestingly, AGE exerts a similar effect on Mfn2 expression in our study, and our results indicate that the change in Mfn2 expression after AGE stimulation is not ROS dependent. Generally, expression of $M f n 2$ is known to be regulated by peroxisome proliferator-activated receptor-coactivater-1a (PGC-1a) (Zorzano et al. 2010). The regulatory mechanism is extremely complicated as it involves gene transcription, translation and posttranslational modification. The precise mechanism by which AGE affects Mfn2 expression needs to be investigated further. To the best of our knowledge, the relationship between AGE and Mfn2 has not been investigated previously. Our study shows that binding of $M f n 2$ to mitochondria, and not Ras binding, mediates the protective effects of AGE stimulation and associated changes in ROS production, collagen IV expression and TFAP2A promoter binding to DN. http://jme.endocrinology-journals.org DOI: 10.1530/JME-16-0031
() 2016 Society for Endocrinology Printed in Great Britain
Published by Bioscientifica Ltd 
Overproduction of collagen IV in DN was previously shown either under high glucose (Tahara et al. 2012) or AGE stimulation in cultured mesangial cells (Abe et al. 2004). In both our previous and present studies, Mfn2 exhibited a protective effect against DN. For instance, overexpression of either wild-type Mfn2 (Tang et al. 2012) or Mfn2-Ras( $\Delta$ ) attenuated the synthesis of collagen IV. In a recent report by Peng and coworkers, without DN limitation, Mfn2 overexpression attenuated hypoxia-induced mitochondrial dysfunction, and the restored mitochondrial morphology subsequently reduced apoptosis in cultured HT22 cells (Peng et al. 2015). Mfn2 overexpression was also found to attenuate injury-induced astrocyte hyperplasia, activation-relevant protein synthesis and cellular proliferation (Liu et al. 2014). In addition, $M f n 2$ overexpression has been shown to suppress the development of atherosclerosis in ApoE (-/-) mice (Guo et al. 2007). However, distinguishing the exact function of $M f n 2$ from others in these reports is difficult. In this study, overexpression of $M f n 2-\operatorname{Ras}(\Delta)$ to compensate for AGE-induced Mfn2 decrease attenuated collagen IV synthesis and subsequently reduced ECM expansion through direct interaction with mitochondria but not in a Ras signal pathway-dependent manner. This strongly supports the idea that $M f n 2$ plays protective roles against DN. Without limitation, the protective role for $M f n 2$ with other functions in DN should be investigated in future studies.

TFs, which are DNA-binding proteins that usually target specific DNA sequences, perform a regulatory role in gene expression. In this study, we found three candidate regions with TFAP2A (transcription factor AP-2 alpha)-like motifs near the rat collagen IV gene TSS. TFAP2A is found in various cellular and viral regulatory complexes and can bind the consensus sequence $5^{\prime}$-GCCNNNGGC-3' to activate the transcription of genes involved in a variety of important biological functions, including proper development of the eye, face, body wall, limb and neural tube (Rada-Iglesias et al. 2012, Hallberg et al. 2014). A previous study demonstrated that various inflammatory cytokines and prostaglandins induce the expression of TFAP2 , which subsequently causes aberrant activation of genes associated with hyperproliferation of mesangial cells and nephrosclerosis, suggesting an important function for TFAP-2 in glomerular disorders (Suyama et al. 2001). In another report, increase in TFAP-2 DNAbinding activity induced by estradiol was found to be involved in collagen metabolism (Guccione et al. 2002).
Consistently, our results revealed that knockdown of TFAP2A by siRNA significantly decreased collagen IV synthesis at the transcriptional level in vitro, indicating that TFAP2A performs a key function in collagen IV gene expression under AGE stimulation. Interestingly, the DNA-binding activity of TFAP2A was suppressed by $M f n 2$ overexpression, which may be responsible for the observed decrease in collagen IV expression after $M f n 2$ transfection under AGE stimulation.

However, the mechanisms for the effect of $M f n 2$ overexpression on TFAP2A binding remain unclear. In this study, we analyzed the epigenetic changes in TFAP2A DNA-binding regions. In general, active promoters are specifically marked by H3K27ac (Rada-Iglesias et al. 2011), which can recruit TFs and thereby activate gene transcription (Spitz \& Furlong 2012). Therefore, we focused on H3K27 acetylation, which is a key step for promoter activation. We observed acetylation changes at the two TFAP2A-binding regions with AGE stimulation or Mfn2 overexpression, which could explain the TFAP2A DNA-binding changes observed previously. Epigenetic modifications and gene expression changes can be induced by changes in metabolite levels because numerous cofactors of chromatin-modifying enzymes are intermediate metabolic products (DeBerardinis \& Thompson 2012, Wellen \& Thompson 2012). For example, acetyl groups for histone acetyltransferases (HAT) originate from fatty acid and glucose metabolism (El-Osta et al. 2008). Mitochondrial functions, including oxidative TCA cycle, membrane potential and ROS production, are closed to histone acetylation (Martinez-Reyes et al. 2015). ROS enhancement of histone acetylation in diabetes or other diseases has also been reported (Bartling \& Drumm 2009, Kabra et al. 2009). Consistent with the results of previous studies, our findings show that ROS performs a key function in histone acetylation. More specifically, ROS affects the acetylation of regulatory elements near the collagen IV TSS. In addition, Mfn 2 transfection reduces mitochondrial ROS production, thereby inhibiting the acetylation of regulatory elements and gene expression. This study is the first to report these effects. However, in our further study in the renal glomeruli of diabetic animals, change in acetylation was only found in Region 1 , but not in Region 2. This result can be explained as follows: (a) the glomerulus is a mixture of numerous types of cells, which results in higher background noise and lower signal intensity associated with acetylation; (b) acetylation at Regions 1 and 2 may be asynchronous

Published by Bioscientifica Ltd. 
and (c) gene regulation in vivo is considerably more complex than that in vitro. Therefore, unraveling the complexity of gene expression regulation in vivo remains a major challenge for future research.

Several questions need to be addressed to fully elucidate the mechanisms underlying the observed results. First, inhibition of $\mathrm{TF}$ binding at regulatory elements partially reduces the expression of collagen IV. This suggests that regulation of collagen IV expression is not restricted to interactions between the regulatory elements and TSS. Second, the interaction between regulatory elements and collagen IV TSS is unclear for technical reasons. Whether direct or indirect interaction is involved requires further investigation. Third, the detailed mechanism by which ROS facilitates histone acetylation is unclear. This may involve an extremely complex unknown molecular network, including localization of HAT, the pioneer TF for acetylation and the recruitment of numerous other cofactors, among others (Smith \& Shilatifard 2014). In conclusion, our results reveal that overexpression of $M f n 2-\operatorname{Ras}(\Delta)$, which has mitochondrial protective function, inhibits the synthesis of collagen IV in DN. Overproduction of ROS by dysfunctional mitochondria affects histone acetylation at regulatory elements near the collagen IV TSS in DN in a cell-specific manner. Furthermore, TF TFAP2A is involved in the mitochondria-ROS-collagen IV pathway through binding to regulatory elements. Therefore, the pathway by which mitochondria affect the function of regulatory elements at promoter regions may be an important area of research for tackling DN. Taken together, our findings improve our understanding of $\mathrm{DN}$ and offer new insights into possible therapeutic targets in $\mathrm{DN}$.

\section{Declaration of interest}

The authors declare that there is no conflict of interest that could be perceived as prejudicing the impartiality of the research reported.

\section{Funding}

This work was supported by the NSFC grant 81270805 , Science and Technology Department of Sichuan province grant 2012 FZ0076 and Chengdu Science and Technology Bureau grant 2015-HM01-00087-SF.

\section{References}

Abe H, Matsubara T, Iehara N, Nagai K, Takahashi T, Arai H, Kita T \& Doi T 2004 Type IV collagen is transcriptionally regulated by Smad1 under advanced glycation end product (AGE) stimulation. Journal of Biological Chemistry 279 14201-14206. (doi:10.1074/jbc. M310427200)
Amiott EA, Lott P, Soto J, Kang PB, McCaffery JM, DiMauro S, Abel ED, Flanigan KM, Lawson VH \& Shaw JM 2008 Mitochondrial fusion and function in Charcot-Marie-Tooth type 2A patient fibroblasts with mitofusin 2 mutations. Experimental Neurology 211 115-127. (doi:10.1016/j.expneurol.2008.01.010)

Anton F, Dittmar G, Langer T \& Escobar-Henriques M 2013 Two deubiquitylases act on mitofusin and regulate mitochondrial fusion along independent pathways. Molecular Cell 49 487-498. (doi:10.1016/j.molcel.2012.12.003)

Atkins RC \& Zimmet P 2010 Diabetic kidney disease: act now or pay later. Kidney International 77 375-377. (doi:10.1038/ki.2009.509)

Bach D, Pich S, Soriano FX, Vega N, Baumgartner B, Oriola J, Daugaard JR, Lloberas J, Camps M, Zierath JR, et al. 2003 Mitofusin-2 determines mitochondrial network architecture and mitochondrial metabolism. A novel regulatory mechanism altered in obesity. Journal of Biological Chemistry 278 17190-17197. (doi:10.1074/jbc. M212754200)

Bailey TL \& Gribskov M 1998 Combining evidence using p-values: application to sequence homology searches. Bioinformatics 14 48-54. (doi:10.1093/bioinformatics/14.1.48)

Bartling TR \& Drumm ML 2009 Oxidative stress causes IL8 promoter hyperacetylation in cystic fibrosis airway cell models. American Journal of Respiratory Cell and Molecular Biology 40 58-65. (doi:10.1165/rcmb.2007-0464OC)

Baumann K 2010 Organelle dynamics: fusing for stability. Nature Reviews Molecular Cell Biology 11 391. (doi:10.1038/nrm2910)

Brosius FC 3rd, Alpers CE, Bottinger EP, Breyer MD, Coffman TM, Gurley SB, Harris RC, Kakoki M, Kretzler M, Leiter EH, et al. 2009 Mouse models of diabetic nephropathy. Journal of the American Society of Nephrology 20 2503-2512. (doi:10.1681/ASN.2009070721)

Brownlee M 2001 Biochemistry and molecular cell biology of diabetic complications. Nature 414 813-820. (doi:10.1038/414813a

Brownlee M 2005 The pathobiology of diabetic complications: a unifying mechanism. Diabetes 54 1615-1625. (doi:10.2337/ diabetes.54.6.1615)

Brownlee M, Cerami A \& Vlassara H 1988 Advanced glycosylation end products in tissue and the biochemical basis of diabetic complications. New England Journal of Medicine 318 1315-1321. (doi:10.1056/NEJM198805193182007)

Chen H, Vermulst M, Wang YE, Chomyn A, Prolla TA, McCaffery JM \& Chan DC 2010a Mitochondrial fusion is required for mtDNA stability in skeletal muscle and tolerance of mtDNA mutations. Cell 141 280-289. (doi:10.1016/j.cell.2010.02.026)

Chen SC, Guh JY, Hwang CC, Chiou SJ, Lin TD, Ko YM, Huang JS, Yang YL \& Chuang LY $2010 b$ Advanced glycation end-products activate extracellular signal-regulated kinase via the oxidative stressEGF receptor pathway in renal fibroblasts. Journal of Cellular Biochemistry 109 38-48. (doi:10.1002/jcb.22376)

Choudhury M, Park PH, Jackson D \& Shukla SD 2010 Evidence for the role of oxidative stress in the acetylation of histone $\mathrm{H} 3$ by ethanol in rat hepatocytes. Alcohol 44 531-540. (doi:10.1016/j. alcohol.2010.06.003)

Coughlan MT, Thorburn DR, Penfold SA, Laskowski A, Harcourt BE, Sourris KC, Tan AL, Fukami K, Thallas-Bonke V, Nawroth PP, et al. 2009 RAGE-induced cytosolic ROS promote mitochondrial superoxide generation in diabetes. Journal of the American Society of Nephrology 20 742-752. (doi:10.1681/ASN.2008050514)

Cui MS, Wang XL, Tang DW, Zhang J, Liu Y \& Zeng SM 2011 Acetylation of $\mathrm{H} 4 \mathrm{~K} 12$ in porcine oocytes during in vitro aging: potential role of ooplasmic reactive oxygen species. Theriogenology $\mathbf{7 5}$ 638-646. (doi:10.1016/j.theriogenology.2010.09.031)

Danaei G, Finucane MM, Lu Y, Singh GM, Cowan MJ, Paciorek CJ, Lin JK, Farzadfar F, Khang YH, Stevens GA, et al. 2011 National, regional, and global trends in fasting plasma glucose and diabetes prevalence since 1980: systematic analysis of health examination surveys and epidemiological studies with 370 country-years and

Published by Bioscientifica Ltd. 
2.7 million participants. Lancet 378 31-40. (doi:10.1016/S01406736(11)60679-X

de Brito OM \& Scorrano L 2009 Mitofusin-2 regulates mitochondrial and endoplasmic reticulum morphology and tethering: the role of Ras. Mitochondrion 9 222-226. (doi:10.1016/j.mito.2009.02.005)

DeBerardinis RJ \& Thompson CB 2012 Cellular metabolism and disease: what do metabolic outliers teach us? Cell 148 1132-1144. (doi:10.1016/j.cell.2012.02.032)

Detmer SA \& Chan DC 2007 Functions and dysfunctions of mitochondrial dynamics. Nature Reviews Molecular Cell Biology $\mathbf{8}$ 870-879. (doi:10.1038/nrm2275)

Dugan LL, You YH, Ali SS, Diamond-Stanic M, Miyamoto S, DeCleves AE, Andreyev A, Quach T, Ly S, Shekhtman G, et al. 2013 AMPK dysregulation promotes diabetes-related reduction of superoxide and mitochondrial function. Journal of Clinical Investigation 123 4888-4899. (doi:10.1172/JCI66218)

El-Osta A, Brasacchio D, Yao D, Pocai A, Jones PL, Roeder RG, Cooper ME \& Brownlee M 2008 Transient high glucose causes persistent epigenetic changes and altered gene expression during subsequent normoglycemia. Journal of Experimental Medicine $\mathbf{2 0 5}$ 2409-2417. (doi:10.1084/jem.20081188)

Fan R, Bonde S, Gao P, Sotomayor B, Chen C, Mouw T, Mouw T, Zavazava N \& Tan K 2012 Dynamic HoxB4-regulatory network during embryonic stem cell differentiation to hematopoietic cells. Blood 119 e139-e147. (doi:10.1182/blood-2011-12-396754)

Grant CE, Bailey TL \& Noble W S 2011 FIMO: scanning for occurrences of a given motif. Bioinformatics 27 1017-1018. (doi:10.1093/ bioinformatics/btr064)

Guccione M, Silbiger S, Lei J \& Neugarten J 2002 Estradiol upregulates mesangial cell MMP-2 activity via the transcription factor AP-2. American Journal of Physiology: Renal Physiology 282 F164-F169. (doi:10.1152/ajprenal.00318.2000)

Guo YH, Chen K, Gao W, Li Q, Chen L, Wang GS \& Tang J 2007 Overexpression of Mitofusin 2 inhibited oxidized low-density lipoprotein induced vascular smooth muscle cell proliferation and reduced atherosclerotic lesion formation in rabbit. Biochemical and Biophysical Research Communications 363 411-417. (doi:10.1016/j. bbrc.2007.08.191)

Hallberg AR, Vorrink SU, Hudachek DR, Cramer-Morales K, Milhem MM, Cornell RA \& Domann FE 2014 Aberrant CpG methylation of the TFAP2A gene constitutes a mechanism for loss of TFAP2A expression in human metastatic melanoma. Epigenetics 9 1641-1647. (doi:10.4161/15592294.2014.988062)

Haneda M, Kikkawa R, Horide N, Togawa M, Koya D, Kajiwara N, Ooshima A \& Shigeta Y 1991 Glucose enhances type IV collagen production in cultured rat glomerular mesangial cells. Diabetologia 34 198-200. (doi:10.1007/BF00418276)

Hernandez-Alvarez MI, Thabit H, Burns N, Shah S, Brema I, Hatunic M, Finucane F, Liesa M, Chiellini C, Naon D, et al. 2010 Subjects with early-onset type 2 diabetes show defective activation of the skeletal muscle PGC-1 \{alpha\}/Mitofusin-2 regulatory pathway in response to physical activity. Diabetes Care 33 645-651. (doi:10.2337/dc09-1305)

Holdsworth SR, Glasgow EF, Atkins RC \& Thomson NM 1978 Cell characteristics of cultured glomeruli from different animal species. Nephron 22 454-459. (doi:10.1159/000181513)

Ide Y, Matsui T, Ishibashi Y, Takeuchi M \& Yamagishi S 2010 Pigment epithelium-derived factor inhibits advanced glycation end productelicited mesangial cell damage by blocking NF-kappaB activation. Microvascular Research 80 227-232. (doi:10.1016/j.mvr.2010.03.015)

Jenuwein T \& Allis CD 2001 Translating the histone code. Science 293 1074-1080. (doi:10.1126/science.1063127)

Jin F, Li Y, Ren B \& Natarajan R 2011 Enhancers: multi-dimensional signal integrators. Transcription 2 226-230. (doi:10.4161/trns.2.5.17712)

Kabra DG, Gupta J \& Tikoo K 2009 Insulin induced alteration in posttranslational modifications of histone $\mathrm{H} 3$ under a hyperglycemic condition in L6 skeletal muscle myoblasts. Biochimica et Biophysica Acta 1792 574-583. (doi:10.1016/j.bbadis.2009.03.003)

Koshiba T, Detmer SA, Kaiser JT, Chen H, McCaffery JM \& Chan DC 2004 Structural basis of mitochondrial tethering by mitofusin complexes. Science 305 858-862. (doi:10.1126/science.1099793)

Kouzarides T 2007 Chromatin modifications and their function. Cell 128 693-705. (doi:10.1016/i.cell.2007.02.005)

Koya D, Hayashi K, Kitada M, Kashiwagi A, Kikkawa R \& Haneda M 2003 Effects of antioxidants in diabetes-induced oxidative stress in the glomeruli of diabetic rats. Journal of the American Society of Nephrology 14 (8 Supplement 3) S250-S253. (doi:10.1097/01. ASN.0000077412.07578.44)

Li YC, Ding XS, Li HM \& Zhang C 2013 Icariin attenuates high glucoseinduced type IV collagen and fibronectin accumulation in glomerular mesangial cells by inhibiting transforming growth factorbeta production and signalling through $\mathrm{G}$ protein-coupled oestrogen receptor 1. Clinical and Experimental Pharmacology and Physiology $\mathbf{4 0}$ 635-643. (doi:10.1111/1440-1681.12143)

Li J, Ke W, Zhou Q, Wu Y, Luo H, Zhou H, Yang B, Guo Y, Zheng Q \& Zhang Y 2014 Tumour necrosis factor-alpha promotes liver ischaemia-reperfusion injury through the PGC-1alpha/Mfn2 pathway. Journal of Cellular and Molecular Medicine 18 1863-1873. (doi:10.1111/jcmm.12320)

Lin Y, Kuro-o M \& Sun Z 2013 Genetic deficiency of anti-aging gene klotho exacerbates early nephropathy in STZ-induced diabetes in male mice. Endocrinology 154 3855-3863. (doi:10.1210/en.20131053)

Liu T, Xue CC, Shi YL, Bai XJ, Li ZF \& Yi CL 2014 Overexpression of mitofusin 2 inhibits reactive astrogliosis proliferation in vitro. Neuroscience Letters 579 24-29. (doi:10.1016/j.neulet.2014.07.002)

Martinez-Reyes I, Diebold LP, Kong H, Schieber M, Huang H, Hensley CT, Mehta MM, Wang T, Santos JH, Woychik R, et al. 2015 TCA cycle and mitochondrial membrane potential are necessary for diverse biological functions. Molecular Cell 61 199-209. (doi:10.1016/j.molcel.2015.12.002)

Mene P, Simonson MS \& Dunn MJ 1989 Physiology of the mesangial cell. Physiological Reviews 69 1347-1424.

Misko A, Jiang S, Wegorzewska I, Milbrandt J \& Baloh RH 2010 Mitofusin 2 is necessary for transport of axonal mitochondria and interacts with the Miro/Milton complex. Journal of Neuroscience $\mathbf{3 0}$ 4232-4240. (doi:10.1523/JNEUROSCI.6248-09.2010)

Moreira JD, Pernomian L, Gomes MS, Pernomian L, Moreira RP, do Prado AF, da Silva CH \& de Oliveira AM 2015 Acute restraint stress increases carotid reactivity in type-I diabetic rats by enhancing Nox4/NADPH oxidase functionality. European Journal of Pharmacology 765 503-516. (doi:10.1016/j.ejphar.2015.09.020)

Oldfield MD, Bach LA, Forbes JM, Nikolic-Paterson D, McRobert A, Thallas V, Atkins RC, Osicka T, Jerums G \& Cooper ME 2001 Advanced glycation end products cause epithelial-myofibroblast transdifferentiation via the receptor for advanced glycation end products (RAGE). Journal of Clinical Investigation 108 1853-1863. (doi:10.1172/JCI11951)

Onozato ML, Tojo A, Goto A, Fujita T \& Wilcox CS 2002 Oxidative stress and nitric oxide synthase in rat diabetic nephropathy: effects of ACEI and ARB. Kidney International 61 186-194. (doi:10.1046/j.1523-1755.2002.00123.x

Papadimitriou A, Peixoto EB, Silva KC, Lopes de Faria JM \& Lopes de Faria JB 2014 Inactivation of AMPK mediates high phosphateinduced extracellular matrix accumulation via NOX4/TGFss-1 signaling in human mesangial cells. Cellular Physiology and Biochemistry 34 1260-1272. (doi:10.1159/000366336)

Park J, Kwon MK, Huh JY, Choi WJ, Jeong LS, Nagai R, Kim WY, Kim J, Lee GT, Lee HB, et al. 2011 Renoprotective antioxidant effect of alagebrium in experimental diabetes. Nephrology Dialysis Transplantation 26 3474-3484. (doi:10.1093/ndt/gfr152) http://jme.endocrinology-journals.org

DOI: 10.1530/JME-16-0031
() 2016 Society for Endocrinology Printed in Great Britain
Published by Bioscientifica Ltd 
Peng C, Rao W, Zhang L, Wang K, Hui H, Wang L, Su N, Luo P, Hao YL, Tu Y, et al. 2015 Mitofusin 2 ameliorates hypoxia-induced apoptosis via mitochondrial function and signaling pathways. International Journal of Biochemistry \& Cell Biology 69 29-40. (doi:10.1016/j. biocel.2015.09.011)

Pozzi A, Zent R, Chetyrkin S, Borza C, Bulus N, Chuang P, Chen D, Hudson B \& Voziyan P 2009 Modification of collagen IV by glucose or methylglyoxal alters distinct mesangial cell functions. Journal of the American Society of Nephrology 20 2119-2125. (doi:10.1681/ ASN.2008080900)

Rada-Iglesias A, Bajpai R, Swigut T, Brugmann SA, Flynn RA \& Wysocka J 2011 A unique chromatin signature uncovers early developmental enhancers in humans. Nature 470 279-283. (doi:10.1038/nature09692)

Rada-Iglesias A, Bajpai R, Prescott S, Brugmann SA, Swigut T \& Wysocka J 2012 Epigenomic annotation of enhancers predicts transcriptional regulators of human neural crest. Cell Stem Cell $\mathbf{1 1}$ 633-648. (doi:10.1016/j.stem.2012.07.006)

Ribaldo PD, Souza DS, Biswas SK, Block K, Lopes de Faria JM \& Lopes de Faria JB 2009 Green tea (Camellia sinensis) attenuates nephropathy by downregulating Nox4 NADPH oxidase in diabetic spontaneously hypertensive rats. Journal of Nutrition 139 96-100. (doi:10.3945/ jn.108.095018)

Sayyed SG, Gaikwad AB, Lichtnekert J, Kulkarni O, Eulberg D, Klussmann S, Tikoo K \& Anders HJ 2010 Progressive glomerulosclerosis in type 2 diabetes is associated with renal histone H3K9 and H3K23 acetylation, H3K4 dimethylation and phosphorylation at serine 10. Nephrology Dialysis Transplantation 25 1811-1817. (doi:10.1093/ndt/gfp730)

Smith E \& Shilatifard A 2014 Enhancer biology and enhanceropathies. Nature Structural \& Molecular Biology 21 210-219. (doi:10.1038/ nsmb.2784)

Soulis-Liparota T, Cooper ME, Dunlop M \& Jerums G 1995 The relative roles of advanced glycation, oxidation and aldose reductase inhibition in the development of experimental diabetic nephropathy in the Sprague-Dawley rat. Diabetologia 38 387-394. (doi:10.1007/BF00410275)

Spitz F \& Furlong EE 2012 Transcription factors: from enhancer binding to developmental control. Nature Reviews Genetics 13 613-626. (doi:10.1038/nrg3207)

Suyama K, Kabuyama Y, Suzuki S, Kawasaki Y, Suzuki J, Suzuki H \& Homma Y 2001 Induction of transcription factor AP-2 by cytokines and prostaglandins in cultured mesangial cells. American Journal of Nephrology 21 307-314. (doi:10.1159/000046266)

Tahara A, Tsukada J, Tomura Y, Yatsu T \& Shibasaki M 2012 Effects of high glucose on AVP-induced hyperplasia, hypertrophy, and type IV collagen synthesis in cultured rat mesangial cells. Endocrine Research 37 216-227. (doi:10.3109/07435800.2012.671400)

Tak E, Ridyard D, Badulak A, Giebler A, Shabeka U, Werner T, Clambey E, Moldovan R, Zimmerman MA, Eltzschig HK, et al. 2013
Protective role for netrin-1 during diabetic nephropathy. Journal of Molecular Medicine 91 1071-1080. (doi:10.1007/s00109-013-1041-1)

Tang WX, Wu WH, Zeng XX, Bo H \& Huang SM 2012 Early protective effect of mitofusion 2 overexpression in STZ-induced diabetic rat kidney. Endocrine 41 236-247. (doi:10.1007/s12020-011-9555-1)

Taye A, Abouzied MM \& Mohafez OM 2013 Tempol ameliorates cardiac fibrosis in streptozotocin-induced diabetic rats: role of oxidative stress in diabetic cardiomyopathy. Naunyn Schmiedebergs Archives of Pharmacology 386 1071-1080. (doi:10.1007/s00210-013-0904-x

Vielhaber S, Debska-Vielhaber G, Peeva V, Schoeler S, Kudin AP, Minin I, Schreiber S, Dengler R, Kollewe K, Zuschratter W, et al. 2013 Mitofusin 2 mutations affect mitochondrial function by mitochondrial DNA depletion. Acta Neuropathologica 125 245-256. (doi:10.1007/s00401-012-1036-y

Wellen KE \& Thompson CB 2012 A two-way street: reciprocal regulation of metabolism and signalling. Nature Reviews Molecular Cell Biology 13 270-276. (doi:10.1038/nrm3305)

Whiteside C, Wang H, Xia L, Munk S, Goldberg HJ \& Fantus IG 2009 Rosiglitazone prevents high glucose-induced vascular endothelial growth factor and collagen IV expression in cultured mesangial cells. Experimental Diabetes Research, 2009910783. (doi:10.1155/2009/910783)

Yan YM, Wang XL, Zhou LL, Zhou FJ, Li R, Tian Y, Zuo ZL, Fang P, Chung AC, Hou FF, et al. 2015 Lingzhilactones from Ganoderma lingzhi ameliorate adriamycin-induced nephropathy in mice. Journal of Ethnopharmacology 176 385-393. (doi:10.1016/j.jep.2015.11.024)

Yu T, Robotham JL \& Yoon Y 2006 Increased production of reactive oxygen species in hyperglycemic conditions requires dynamic change of mitochondrial morphology. PNAS 103 2653-2658. (doi:10.1073/pnas.0511154103)

Zhan M, Usman IM, Sun L \& Kanwar YS 2014 Disruption of renal tubular mitochondrial quality control by myo-inositol oxygenase in diabetic kidney disease. Journal of the American Society of Nephrology. (doi:10.1681/ASN.2014050457)

Zhang XH, Zheng B, Han M, Miao SB \& Wen JK 2009 Synthetic retinoid Am80 inhibits interaction of KLF5 with RAR alpha through inducing KLF5 dephosphorylation mediated by the PI3K/Akt signaling in vascular smooth muscle cells. FEBS Letters 583 1231-1236. (doi:10.1016/j.febslet.2009.03.016)

Zhou VW, Goren A \& Bernstein BE 2011 Charting histone modifications and the functional organization of mammalian genomes. Nature Reviews Genetics 12 7-18. (doi:10.1038/nrg2905)

Zorzano A, Hernandez-Alvarez MI, Palacin M \& Mingrone G 2010 Alterations in the mitochondrial regulatory pathways constituted by the nuclear co-factors PGC-1alpha or PGC-1beta and mitofusin 2 in skeletal muscle in type 2 diabetes. Biochimica et Biophysica Acta 1797 1028-1033. (doi:10.1016/j.bbabio.2010.02.017)

Received in final form 23 September 2016

Accepted 3 October 2016 http://jme.endocrinology-journals.org

DOI: 10.1530/JME-16-0031
๑) 2016 Society for Endocrinology Printed in Great Britain
Published by Bioscientifica Ltd 